Louisiana State University

LSU Digital Commons

Faculty Publications

Department of Physics \& Astronomy

$12-10-2012$

\title{
THE chara array angular diameter of HR 8799 favors planetary masses for its imaged companions
}

\author{
Ellyn K. Baines \\ Naval Research Laboratory \\ Russel J. White \\ Georgia State University \\ Daniel Huber \\ NASA Ames Research Center \\ Jeremy Jones \\ Georgia State University \\ Tabetha Boyajian \\ Georgia State University
}

See next page for additional authors

Follow this and additional works at: https://digitalcommons.Isu.edu/physics_astronomy_pubs

\section{Recommended Citation}

Baines, E., White, R., Huber, D., Jones, J., Boyajian, T., McAlister, H., Ten Brummelaar, T., Turner, N., Sturmann, J., Sturmann, L., Goldfinger, P., Farrington, C., Riedel, A., Ireland, M., Von Braun, K., \& Ridgway, S. (2012). THE chara array angular diameter of HR 8799 favors planetary masses for its imaged companions. Astrophysical Journal, 761 (1) https://doi.org/10.1088/0004-637X/761/1/57

This Article is brought to you for free and open access by the Department of Physics \& Astronomy at LSU Digital Commons. It has been accepted for inclusion in Faculty Publications by an authorized administrator of LSU Digital Commons. For more information, please contact ir@lsu.edu. 


\section{Authors}

Ellyn K. Baines, Russel J. White, Daniel Huber, Jeremy Jones, Tabetha Boyajian, Harold A. McAlister, Theo A. Ten Brummelaar, Nils H. Turner, Judit Sturmann, Laszlo Sturmann, P. J. Goldfinger, Christopher D.

Farrington, Adric R. Riedel, Michael Ireland, Kaspar Von Braun, and Stephen T. Ridgway 


\title{
THE CHARA ARRAY ANGULAR DIAMETER OF HR 8799 FAVORS PLANETARY MASSES FOR ITS IMAGED COMPANIONS
}

\author{
Ellyn K. Baines ${ }^{1}$, Russel J. White ${ }^{2}$, Daniel Huber ${ }^{3}$, Jeremy Jones $^{2}$, Tabetha Boyajian $^{2}$, Harold A. McAlister ${ }^{2}$, \\ Theo A. Ten BrummelaAR ${ }^{2}$, Nils H. Turner ${ }^{2}$, Judit Sturmann ${ }^{2}$, Laszlo Sturmann ${ }^{2}$, P. J. Goldfinger ${ }^{2}$, \\ Christopher D. Farrington ${ }^{2}$, Adric R. Riedel ${ }^{2}$, Michael Ireland ${ }^{4}$, Kaspar von Braun $^{5}$, And Stephen T. Ridgway ${ }^{6}$ \\ ${ }^{1}$ Remote Sensing Division, Naval Research Laboratory, 4555 Overlook Avenue SW, Washington, DC 20375, USA; ellyn.baines@nrl.navy.mil \\ ${ }^{2}$ Center for High Angular Resolution Astronomy, Georgia State University, P.O. Box 3969, Atlanta, GA 30302-3969, USA \\ ${ }^{3}$ NASA Ames Research Center, Moffett Field, CA 94035, USA \\ ${ }^{4}$ Department of Physics \& Astronomy, Macquarie University, New South Wales, NSW 2109, Australia \\ ${ }^{5}$ NASA Exoplanet Science Institute, California Institute of Technology, 770 S. Wilson Ave., MS 100-22, Pasadena, CA 91125-2200, USA \\ ${ }^{6}$ Kitt Peak National Observatory, National Optical Astronomy Observatory, P.O. Box 26732, Tucson, AZ 85726-6732, USA \\ Received 2012 July 3; accepted 2012 September 29; published 2012 November 21
}

\begin{abstract}
HR 8799 is an hF0 mA5 $\gamma$ Doradus-, $\lambda$ Bootis-, Vega-type star best known for hosting four directly imaged candidate planetary companions. Using the CHARA Array interferometer, we measure HR 8799's limb-darkened angular diameter to be $0.342 \pm 0.008$ mas (an error of only 2\%). By combining our measurement with the star's parallax and photometry from the literature, we greatly improve upon previous estimates of its fundamental parameters, including stellar radius $\left(1.44 \pm 0.06 R_{\odot}\right)$, effective temperature (7193 $\pm 87 \mathrm{~K}$, consistent with F0), luminosity $\left(5.05 \pm 0.29 L_{\odot}\right)$, and the extent of the habitable zone (HZ; 1.62-3.32 AU). These improved stellar properties permit much more precise comparisons with stellar evolutionary models, from which a mass and age can be determined, once the metallicity of the star is known. Considering the observational properties of other $\lambda$ Bootis stars and the indirect evidence for youth of HR 8799, we argue that the internal abundance, and what we refer to as the effective abundance, is most likely near solar. Finally, using the Yonsei-Yale evolutionary models with uniformly scaled solar-like abundances, we estimate HR 8799's mass and age considering two possibilities: $1.516_{-0.024}^{+0.038} M_{\odot}$ and $33_{-13.2}^{+7} \mathrm{Myr}$ if the star is contracting toward the zero-age main sequence or $1.513_{-0.024}^{+0.023} M_{\odot}$ and $90_{-50}^{+381} \mathrm{Myr}$ if it is expanding from it. This improved estimate of HR 8799's age with realistic uncertainties provides the best constraints to date on the masses of its orbiting companions, and strongly suggests they are indeed planets. They nevertheless all appear to orbit well outside the $\mathrm{HZ}$ of this young star.
\end{abstract}

Key words: planetary systems - stars: fundamental parameters - stars: individual (HR 8799) - techniques: high angular resolution - techniques: interferometric

Online-only material: color figures, machine-readable table

\section{INTRODUCTION}

HR 8799 (HD 218396, HIP 114189) is a $\gamma$ Doradus-type star, a class characterized by pulsations on timescales longer than the typical $\delta$ Scuti stars, their neighbors on the Hertzsprung-Russell (H-R) diagram (Breger 1979; Zerbi et al. 1999; Kaye et al. 1999; Handler 1999). HR 8799 is also a $\lambda$ Bootis-type star, which is a spectroscopically defined group of non-magnetic, chemically peculiar, Population I A- and early F-type stars that show metal deficiencies in Fe-peak elements while showing solar to slightly over solar abundances of $\mathrm{C}, \mathrm{N}, \mathrm{O}$, and $\mathrm{S}$ (Venn \& Lambert 1990; Paunzen et al. 1998; Gray \& Kaye 1999). In addition, HR 8799 is a "Vega-like" star, possessing excess infrared emission longward of $\sim 20 \mu \mathrm{m}$ (Sadakane \& Nishida 1986; Su et al. 2009), attributed to thermal emission from a debris disk.

Aside from these three distinguishing characteristics, interest in HR 8799 was amplified in 2008 with the discovery of three planet-like companions at projected separations of 24,38 , and $68 \mathrm{AU}$ (Marois et al. 2008). From comparisons of the observed fluxes to the predictions of evolutionary models, Marois et al. estimated companion masses of 5-11 $M_{\text {Jupiter }}$ for companion b and 7-13 $M_{\text {Jupiter }}$ for companions $\mathrm{c}$ and d, each. Because the masses of the companions depend on the age of the system, Marois et al. used four lines of reasoning to estimate the host star's age: (1) comparing the star's galactic space motion to other young stars in the solar neighborhood, (2) placing the star on a color-magnitude diagram, (3) characterizing the typical ages of $\lambda$ Boo and $\gamma$ Dor stars, and (4) the assumption that HR 8799 has a massive debris disk, which is typically only found to be associated with young stars $(\lesssim 500 \mathrm{Myr})$. Based on these characteristics, they constrained an age range between 30 and $160 \mathrm{Myr}$. A fourth companion was imaged two years later with an estimated mass of $7_{-2}^{+3} M_{\text {Jupiter }}$ if the system is $30 \mathrm{Myr}$ old and $10 \pm 3 M_{\text {Jupiter }}$ if $60 \mathrm{Myr}$ old (Marois et al. 2010).

Marley et al. (2012) fit atmospheric and evolution models to the data to determine the masses, radii, temperatures, gravities, and cloud properties for the planets. They found masses of 26 $M_{\text {Jupiter }}$ for planet b and 8-11 $M_{\text {Jupiter }}$ for planets c and d, though they acknowledge that their model fit for b's mass is not likely to be the true value. They also determined an age range of $360 \mathrm{Myr}$, 40-100 My, and 30-100 Myr for each planet, respectively.

The ages adopted by Marois et al. $(2008,2010)$ have become a topic of some debate. The star's age is of vital interest because it is directly linked to the masses of the companions. If the star is young, its companions are brighter and their inferred masses are lower-i.e., planets - than if the star is older, in which case its more massive companions-i.e., brown dwarfs-have cooled significantly and could be mistaken for planets (Moro-Martín et al. 2010). For example, if HR 8799 is as old as the Hyades (625 Myr; Perryman et al. 1998), all companions would be 
Table 1

HR 8799 System Parameters from the Literature

\begin{tabular}{|c|c|c|c|}
\hline $\begin{array}{l}M_{\text {star }} \\
\left(M_{\odot}\right)\end{array}$ & $\begin{array}{c}\text { Age } \\
(\mathrm{Myr})\end{array}$ & Method Used & Reference \\
\hline $1.47 \pm 0.30$ & $\ldots$ & Spectral synthesis \& spectrophotometry & Gray \& Kaye (1999) \\
\hline$\ldots$ & $50-1128$ & H-R diagram placement & Song et al. (2001) \\
\hline$\ldots$ & 30 & Stellar kinematics, H-R diagram placement vs. isochrones & Zuckerman \& Song (2004) \\
\hline$\ldots$ & $20-150$ & Local Association membership & Moór et al. (2006) \\
\hline . & 590 & IR excess & Chen et al. (2006) \\
\hline$\ldots$ & 30 & IR excess, H-R diagram placement & Rhee et al. (2007) \\
\hline 1.5 & $30-160$ & Multiple methods & Marois et al. (2008) \\
\hline $1.2-1.6$ & $\lesssim 100 \mathrm{Myr}$ & Dynamical stability analysis & Goździewski \& Migaszewski (2009) \\
\hline$\ldots$ & $\lesssim 50$ & Observational data analysis & Reidemeister et al. (2009) \\
\hline 1.5 & $\lesssim 100 \mathrm{Myr}$ & Dynamical stability analysis & Fabrycky \& Murray-Clay (2010) \\
\hline$\ldots$ & $\sim 100 \mathrm{Myr}$ & Disk inclination & Moro-Martín et al. (2010) \\
\hline $1.32-1.33^{\mathrm{a}}$ & $1123-1623$ & Asteroseismology & Moya et al. (2010a) \\
\hline $1.44-1.45^{\mathrm{b}}$ & $26-430$ & Asteroseismology & Moya et al. (2010a) \\
\hline 1.32 & $1126-1486$ & Asteroseismology & Moya et al. (2010a) \\
\hline$\ldots$ & 30 & Columba Association membership & Doyon et al. (2010) \\
\hline$\ldots$ & 30 & Columba Association membership & Zuckerman et al. (2011) \\
\hline$\ldots$ & $30-100$ & Direct imaging & Currie et al. (2011) \\
\hline$\cdots$ & $30-300$ & Atmospheric/evolution model fitting & Marley et al. (2012) \\
\hline$\ldots$ & $30-155$ & Dynamical stability analysis & Sudol \& Haghighipour (2012) \\
\hline
\end{tabular}

Notes.

a Solution 1 .

b Solution 2 .

Table 2

Calibrator Star Properties

\begin{tabular}{lccccc}
\hline \hline HD & $\begin{array}{c}\text { Spectral } \\
\text { Type }\end{array}$ & $\begin{array}{c}V \\
(\mathrm{mag})\end{array}$ & $\begin{array}{c}K \\
(\mathrm{mag})\end{array}$ & $E(B-V)$ & $\begin{array}{c}\theta_{\text {photometric }} \\
(\mathrm{mas})\end{array}$ \\
\hline 213617 & F1 V & 6.42 & $5.58 \pm 0.02$ & 0.03 & $0.288 \pm 0.006$ \\
214698 & A2 V & 6.33 & $6.21 \pm 0.02$ & 0.05 & $0.189 \pm 0.005$ \\
219487 & F5 V & 6.60 & $5.54 \pm 0.02$ & 0.05 & $0.304 \pm 0.006$
\end{tabular}

Notes. Spectral types are from SIMBAD; $V$ magnitudes are from Mermilliod (1991) except for HD 214698, which is from Perryman \& ESA (1997). No errors were listed so we assigned errors of \pm 0.01 mag; $K$ magnitudes are from Cutri et al. (2003).

more massive than $13.6 M_{\text {Jupiter }}$, and thus more appropriately called brown dwarfs according to popular convention. One example of the effect of presumed ages on masses involved the announcement by Lafrenière et al. (2010) of a directly imaged planetary companion to 1RXS J160929.1-210524 in the Upper Scorpius association, which relied on an assumed cluster age of 5 Myr. Soon afterward, Pecaut et al. (2012) determined an older age for the cluster: $11_{-2}^{+1}$ Myr. In this case, the imaged companion has a mass of $\sim 14 M_{\text {Jupiter }}$, putting it just above the planetary mass limit.

Moya et al. (2010a) refute the Marois et al. (2008) young age of HR 8799 on all four counts. They point out that (1) space motions of young disk stars are often inconclusive, (2) the uncertain internal metallicity of HR 8799 makes comparisons with evolutionary models and other stellar clusters unreliable, (3) that $\lambda$ Boo and $\gamma$ Dor type stars have a broad range of ages, and (4) that debris disks are "highly chaotic and unpredictable," and cannot be used to estimate stellar age. Moya et al. (2010a) instead determined HR 8799's age by modeling $\gamma$ Dor pulsations detected by Zerbi et al. (1999) and adopting spectroscopic parameters by Allende Prieto \& Lambert (1999). Although primarily limited by the unknown inclination of the
Table 3

Calibrated Visibilities

\begin{tabular}{|c|c|c|c|c|}
\hline Date & $\begin{array}{c}\text { Calibrator } \\
\text { Used }\end{array}$ & $\begin{array}{l}\text { Spatial Freq } \\
\left(10^{8} \mathrm{rad}^{-1}\right)\end{array}$ & $V^{2}$ & $\sigma V^{2}$ \\
\hline \multirow[t]{23}{*}{2010 Aug 25} & HD 213617 & 2.223 & 0.861 & 0.081 \\
\hline & & 2.245 & 0.768 & 0.069 \\
\hline & & 2.267 & 0.785 & 0.069 \\
\hline & & 2.289 & 0.807 & 0.076 \\
\hline & & 2.311 & 0.804 & 0.089 \\
\hline & & 2.332 & 0.729 & 0.094 \\
\hline & & 2.353 & 0.811 & 0.138 \\
\hline & & 2.375 & 0.844 & 0.146 \\
\hline & & 2.397 & 0.924 & 0.142 \\
\hline & & 2.419 & 0.976 & 0.135 \\
\hline & & 2.442 & 0.892 & 0.092 \\
\hline & & 2.464 & 0.834 & 0.066 \\
\hline & & 2.486 & 0.788 & 0.059 \\
\hline & & 2.507 & 0.764 & 0.057 \\
\hline & & 2.528 & 0.804 & 0.063 \\
\hline & & 2.550 & 0.742 & 0.059 \\
\hline & & 2.571 & 0.728 & 0.067 \\
\hline & & 2.593 & 0.713 & 0.084 \\
\hline & & 2.613 & 0.771 & 0.104 \\
\hline & & 2.634 & 0.840 & 0.120 \\
\hline & & 2.655 & 0.784 & 0.103 \\
\hline & & 2.677 & 0.827 & 0.096 \\
\hline & & 2.699 & 0.801 & 0.077 \\
\hline
\end{tabular}

Notes. All data were taken using the S2-W2 baseline (177 m).

(This table is available in its entirety in a machine-readable form in the online journal. A portion is shown here for guidance regarding its form and content.)

star's rotation axis, they find a preferred age of between 1.1 and 1.5 Gyr; again, this age implies that the companions to HR 8799 are brown dwarfs.

Moro-Martín et al. (2010) subsequently challenged the claim made by Moya et al. (2010a), finding an age of the system 
Table 4

HR 8799 Angular Diameter Measurements

\begin{tabular}{|c|c|c|c|}
\hline Date & $\begin{array}{c}\text { Calibrator } \\
\text { HD }\end{array}$ & $\begin{array}{c}\theta_{\mathrm{UD}} \\
(\mathrm{mas})\end{array}$ & $\begin{array}{c}\theta_{\mathrm{LD}} \\
(\mathrm{mas})\end{array}$ \\
\hline \multirow[t]{3}{*}{2010 Aug 25} & 213617 & $0.297 \pm 0.021$ & $0.309 \pm 0.021$ \\
\hline & 219487 & $0.381 \pm 0.015$ & $0.397 \pm 0.016$ \\
\hline & All & $0.357 \pm 0.016$ & $0.372 \pm 0.016$ \\
\hline \multirow[t]{3}{*}{2010 Sep 7} & 213617 & $0.342 \pm 0.015$ & $0.356 \pm 0.014$ \\
\hline & 219487 & $0.375 \pm 0.010$ & $0.391 \pm 0.010$ \\
\hline & All & $0.359 \pm 0.009$ & $0.373 \pm 0.010$ \\
\hline \multirow[t]{3}{*}{2010 Sep 8} & 213617 & $0.302 \pm 0.018$ & $0.315 \pm 0.019$ \\
\hline & 219487 & $0.313 \pm 0.012$ & $0.326 \pm 0.013$ \\
\hline & All & $0.308 \pm 0.011$ & $0.321 \pm 0.012$ \\
\hline \multirow[t]{4}{*}{2011 Oct 20} & 213617 & $0.320 \pm 0.013$ & $0.333 \pm 0.014$ \\
\hline & 214698 & $0.349 \pm 0.017$ & $0.363 \pm 0.018$ \\
\hline & 219487 & $0.314 \pm 0.015$ & $0.327 \pm 0.015$ \\
\hline & All & $0.331 \pm 0.009$ & $0.345 \pm 0.010$ \\
\hline \multirow[t]{4}{*}{2011 Oct 21} & 213617 & $0.336 \pm 0.010$ & $0.350 \pm 0.010$ \\
\hline & 214698 & $0.320 \pm 0.012$ & $0.333 \pm 0.013$ \\
\hline & 219487 & $0.332 \pm 0.016$ & $0.346 \pm 0.017$ \\
\hline & All & $0.330 \pm 0.019$ & $0.343 \pm 0.021$ \\
\hline \multirow[t]{4}{*}{2011 Oct 22} & 213617 & $0.308 \pm 0.010$ & $0.321 \pm 0.011$ \\
\hline & 214698 & $0.308 \pm 0.014$ & $0.320 \pm 0.015$ \\
\hline & 219487 & $0.332 \pm 0.008$ & $0.346 \pm 0.009$ \\
\hline & All & $0.328 \pm 0.023$ & $0.341 \pm 0.022$ \\
\hline 2011 Sep 30 & 214698 & $0.323 \pm 0.009$ & $0.338 \pm 0.008$ \\
\hline All dates & 213617 & $0.327 \pm 0.008$ & $0.341 \pm 0.008$ \\
\hline 2011 Aug data only & & $0.326 \pm 0.008$ & $0.340 \pm 0.008$ \\
\hline All dates & 214698 & $0.327 \pm 0.009$ & $0.341 \pm 0.010$ \\
\hline All dates & 219487 & $0.343 \pm 0.008$ & $0.358 \pm 0.008$ \\
\hline 2011 Aug data only & & $0.328 \pm 0.009$ & $0.342 \pm 0.009$ \\
\hline All dates & All & $0.333 \pm 0.007$ & $0.347 \pm 0.007$ \\
\hline Final fit: 2011 data & All & $0.327 \pm 0.007$ & $0.342 \pm 0.008$ \\
\hline
\end{tabular}

Notes. Figure 10 shows a graphical view of these values. Data were obtained using the S2-W2 baseline at $177 \mathrm{~m}$ for all nights except for $2011 \mathrm{Sep} 30$, which were obtained using the $\mathrm{S} 1-\mathrm{E} 1$ baseline at $331 \mathrm{~m}$.

Table 5

HR 8799 Photometry

\begin{tabular}{lcc}
\hline \hline Band & $\begin{array}{c}\text { Average } \\
(\mathrm{mag})\end{array}$ & $\begin{array}{c}\text { No. of } \\
\text { Measurements }\end{array}$ \\
\hline$U$ & $6.191 \pm 0.016$ & 2 \\
$B$ & $6.235 \pm 0.016$ & 2 \\
$V$ & $5.980 \pm 0.016$ & 2 \\
$u$ & $7.471 \pm 0.025$ & 7 \\
$v$ & $6.468 \pm 0.025$ & 7 \\
$b$ & $6.143 \pm 0.027$ & 7 \\
$y$ & $5.962 \pm 0.026$ & 7 \\
$J$ & $5.383 \pm 0.027$ & 1 \\
$H$ & $5.280 \pm 0.018$ & 1 \\
$K$ & $5.240 \pm 0.018$ & 1 \\
\hline
\end{tabular}

of $\sim 100 \mathrm{Myr}$ after considering the limiting case for orbital stability; at these relatively small separations, massive companions would not be dynamically stable on long timescales. They used the double 4:2:1 mean motion resonance configuration presented by other authors (e.g., Reidemeister et al. 2009; Goździewski \& Migaszewski 2009; Fabrycky \& Murray-Clay 2010) as a way to ensure dynamical stability by avoiding close encounters. Moro-Martín et al. used evolutionary models to predict ages from 150 to $350 \mathrm{Myr}$ and conclude that the dynamical state of the system favors an age of $\sim 100 \mathrm{Myr}$, which places the companions just shy of the brown dwarf regime. However, they
Table 6

HR 8799 Stellar Parameters

\begin{tabular}{|c|c|c|}
\hline Parameter & Value & Reference \\
\hline$V$ magnitude & $5.98 \pm 0.01$ & Mermilliod (1991) \\
\hline$K$ magnitude & $5.24 \pm 0.02$ & Cutri et al. (2003) \\
\hline$\pi$ (mas) & $25.38 \pm 0.70$ & van Leeuwen (2007) \\
\hline Distance (pc) & $39.40 \pm 1.09$ & van Leeuwen (2007) \\
\hline$\mu_{\lambda}$ & $0.49 \pm 0.02$ & Claret \& Bloemen (2011) \\
\hline$A_{\mathrm{V}}$ & $0.00 \pm 0.01$ & This work; PED fit \\
\hline$F_{\mathrm{BOL}}\left(10^{-7} \mathrm{erg} \mathrm{s}^{-1} \mathrm{~cm}^{-2}\right)$ & $1.043 \pm 0.012$ & This work; PED fit \\
\hline$T_{\text {eff,estimated }}(\mathrm{K})$ & $7211 \pm 90$ & This work; PED fit \\
\hline$\theta_{\text {UD }}($ mas $)$ & $0.327 \pm 0.008$ & This work \\
\hline$\theta_{\mathrm{LD}}(\mathrm{mas})$ & $0.342 \pm 0.008$ & This work \\
\hline$R_{\text {linear }}\left(R_{\odot}\right)$ & $1.44 \pm 0.06$ & This work \\
\hline$T_{\text {eff }}(\mathrm{K})$ & $7193 \pm 87$ & This work \\
\hline$L\left(L_{\odot}\right)$ & $5.05 \pm 0.29$ & This work \\
\hline \multicolumn{3}{|c|}{ If the star is contracting toward the ZAMS } \\
\hline Age (Myr) & $33_{-13.2}^{+7}$ & This work \\
\hline$\underline{\operatorname{Mass}}\left(M_{\odot}\right)$ & $1.516_{-0.024}^{+0.038}$ & This work \\
\hline \multicolumn{3}{|c|}{ If the star is expanding from the ZAMS } \\
\hline Age (Myr) & $90_{-50}^{+381}$ & This work \\
\hline $\operatorname{Mass}\left(M_{\odot}\right)$ & $1.513_{-0.024}^{+0.023} M_{\odot}$ & This work \\
\hline
\end{tabular}

point out that the average temperature of $7350 \mathrm{~K}$ and luminosity of $5 L_{\odot}$ from Sadakane (2006) and Moya et al. (2010b) imply an age for the star that is very young when compared to other $\lambda$ Boo stars.

In addition to these recent attempts to estimate HR 8799's age, many other efforts have been made to determine its age in the last decade. The resulting values vary from $20 \mathrm{Myr}$ up to 1.5 Gyr. In Table 1, we summarize both the attempts described above and other efforts. A variety of methods were utilized, including inspecting the star's proper motion, comparing its position on an H-R diagram with isochrones, inspecting the star for infrared excess, asteroseismology, astrometry, spectroscopy, dynamical stability analysis, disk symmetry, direct imaging, and group membership.

Here, we seek to greatly improve our understanding of HR 8799's age by using interferometric observations to directly measure its angular diameter. This value, when combined with the HIPPARCOS parallax, yields the star's physical radius. In combination with HR 8799's bolometric flux determined from broadband photometry, the stellar luminosity and effective temperature are precisely determined. Using these results, we provide new age and mass estimates based on comparisons with the stellar evolutionary models, adopting a metallicity most appropriate for $\lambda$ Boo stars and HR 8799 in particular. Specifically, Section 2 describes the interferometric observations and calibrator star selection; Section 3 discusses the visibility measurements and how stellar parameters were calculated, including angular diameter, radius, luminosity, and temperature; Section 4 explores the physical implications of the interferometric observations; and Section 5 summarizes our findings.

\section{INTERFEROMETRIC OBSERVATIONS}

Observations were obtained using the Center for High Angular Resolution Astronomy (CHARA) Array, a six element optical-infrared interferometer located on Mount Wilson, California (ten Brummelaar et al. 2005). All observations used 


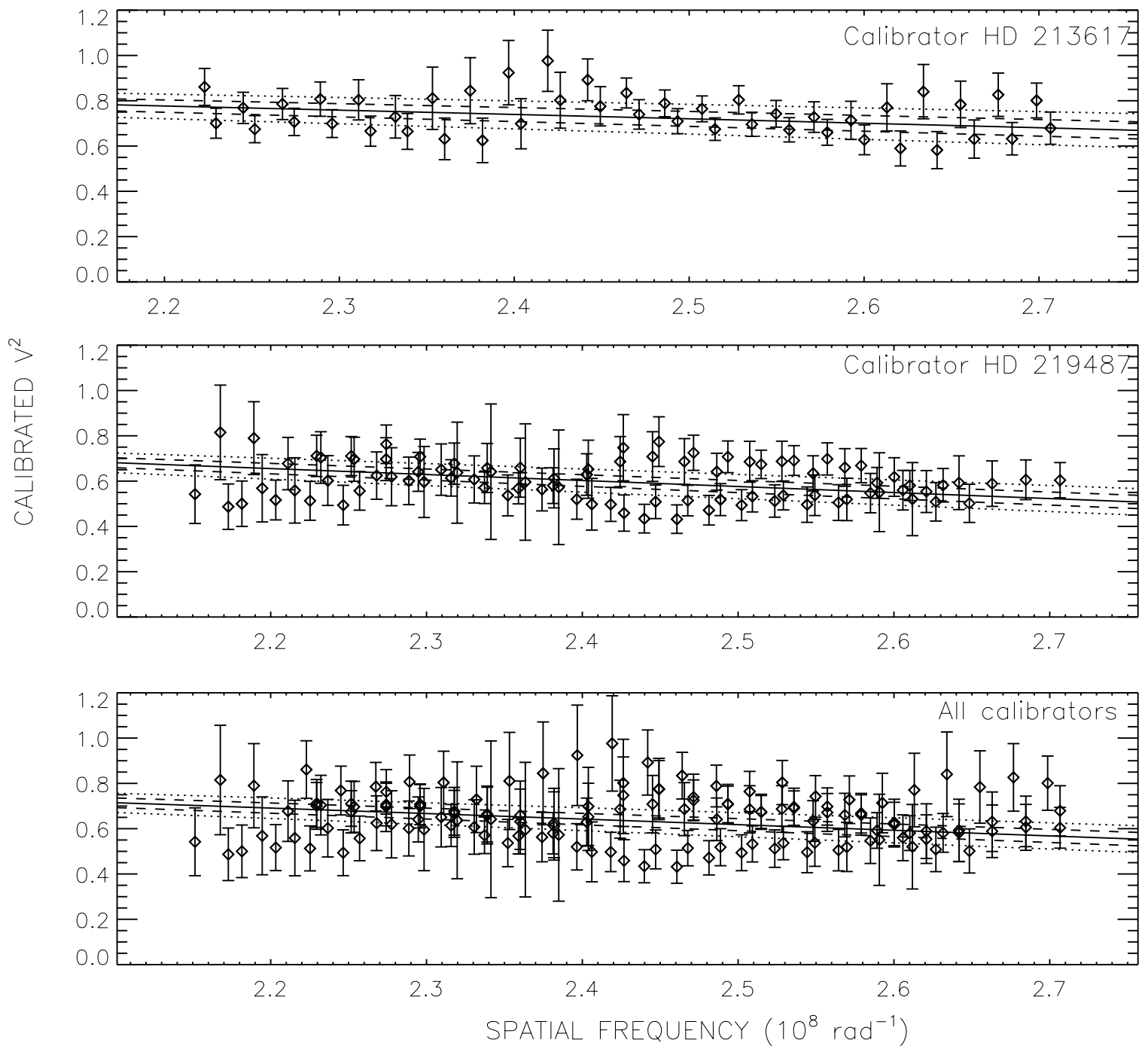

Figure 1. $\theta_{\mathrm{LD}}$ fit for HR 8799 on 2010 August 25 . The diamonds and vertical lines are the measured visibilities and their associated errors, the solid line is the best-fit $\mathrm{LD}$ diameter, the dashed line is the $1 \sigma$ error, and the dotted line is the $2 \sigma$ error.

the Precision Astronomical Visible Observations (PAVO) beam combiner with a spectral bandpass of $\sim 650-800 \mathrm{~nm}$. For a description of the PAVO instrument and data reduction procedure, see Ireland et al. (2008). We observed HR 8799 over seven nights spanning two years with the S2-W2 and S1-E1 telescope pairs with maximum baselines of $177 \mathrm{~m}$ and $331 \mathrm{~m}$, respectively. ${ }^{7}$

We interleaved calibrator star and HR 8799 observations so that every target was flanked by calibrator observations made as close in time as possible, which allowed us to convert instrumental target and calibrator visibilities to calibrated visibilities for the target. Calibrators are stars with predicted diameters that are significantly smaller and in close proximity in the sky to the target star. Reliable calibrators were chosen to be single stars with angular diameter estimates $\lesssim 0.3$ mas so they were nearly unresolved on the baseline used, which meant uncertainties in each calibrator's diameter did not affect the target's diameter calculation as much as if the calibrator star had a larger angular size.

\footnotetext{
7 The three arms of the CHARA Array are denoted by their cardinal directions: "S," "E," and "W" are south, east, and west, respectively. Each arm bears two telescopes, numbered " 1 " for the telescope farthest from the beam combining laboratory and " 2 " for the telescope closer to the lab. The "baseline" is the distance between the telescopes.
}

In order to estimate the reddening of each calibrator star, we obtained the Tycho $\left(B_{\mathrm{T}}-V_{\mathrm{T}}\right)$ color from Perryman \& ESA (1997) and converted to $(B-V)$ using the table in Bessell (2000). We then compared the observed $(B-V)$ value with the list of intrinsic $(B-V)$ colors as a function of spectral type given by Schmidt-Kaler $(1982)^{8}$ to arrive at an estimate of $E(B-V)$, and adopted the reddening law described in O'Donnell (1994) to deredden the observed magnitudes. The photometric angular diameters were then determined using the relationship between the $(V-K)$ color and $\log \theta_{\mathrm{LD}}$ from Kervella et al. (2004). The error in the diameters is due to the relative calibration error stated in the Kervella et al. (2004) paper as well as errors in photometry measurements. Table 2 lists the input photometry and resulting photometrically estimated angular diameters of the calibrator stars.

\section{RESULTS}

\subsection{Angular Diameter Measurement}

The observed quantity of an interferometer is fringe contrast or "visibility," more formally defined as the correlation of two

8 The table was obtained from http://obswww.unige.ch/gcpd/mk01bv.html 

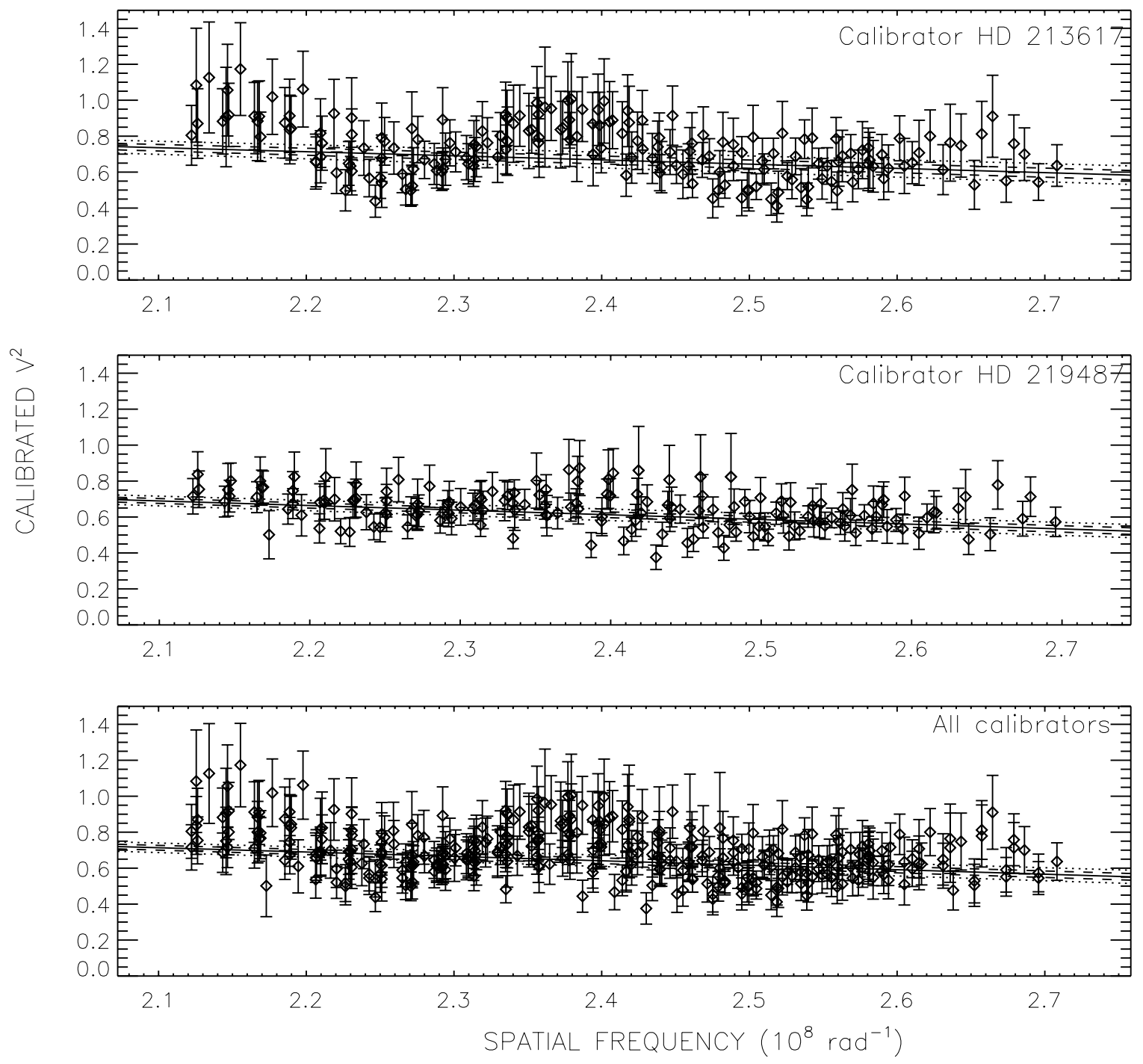

Figure 2. $\theta_{\text {LD }}$ fit for HR 8799 on 2010 September 7. The symbols are the same as in Figure 1.

wave fronts whose amplitude is the visibility squared $\left(V^{2}\right)$, which is fit to a model of a uniformly illuminated disk (UD) that represents the face of the star. Diameter fits to $V^{2}$ were based upon the UD approximation given by $V^{2}=\left(\left[2 J_{1}(x)\right] / x\right)^{2}$, where $J_{1}$ is the first-order Bessel function and $x=\pi B \theta_{\mathrm{UD}} \lambda^{-1}$, where $B$ is the projected baseline at the star's position, $\theta_{\mathrm{UD}}$ is the apparent UD angular diameter of the star, and $\lambda$ is the effective wavelength of the observation (Shao \& Colavita 1992). A more realistic model of a star's disk involves limb-darkening (LD), and the relationship incorporating the linear LD coefficient $\mu_{\lambda}$ (Hanbury Brown et al. 1974) is

$$
\begin{aligned}
V^{2}= & \left(\frac{1-\mu_{\lambda}}{2}+\frac{\mu_{\lambda}}{3}\right)^{-2} \\
& \times\left[\left(1-\mu_{\lambda}\right) \frac{J_{1}(x)}{x}+\mu_{\lambda}\left(\frac{\pi}{2}\right)^{1 / 2} \frac{J_{3 / 2}(x)}{x^{3 / 2}}\right]^{2} .
\end{aligned}
$$

Table 3 lists the date of observation, the calibrator used, $\lambda / B$, the calibrated visibilities $\left(V^{2}\right)$, and errors in $V^{2}\left(\sigma V^{2}\right)$.

The LD coefficient was obtained from Claret \& Bloemen (2011) after adopting the effective temperature $\left(T_{\text {eff }}\right)$ and surface gravity $(\log g$ ) values from Allende Prieto \& Lambert (1999), which were $7586 \mathrm{~K}$ and 4.35 , respectively. The resulting UD and
LD angular diameters are listed in Table 6. Figures 1-8 show the LD diameter fits for HR 8799 by night and by calibrator and Figure 9 shows all the data combined.

Table 4 lists the resulting angular diameter fits for each night using each calibrator, and Figure 10 shows a graphical version of the results. Though there is some scatter in the diameter fit from each night/calibrator combination, the scatter from the 2011 data is less pronounced than from 2010 data, which is seen in the diameter fits shown in Figures 4-7. In particular, for observations obtained in 2010, the data exhibit sinusoidal-like variations about the best angular diameter fit; the variations are not seen in 2011 data. This is almost certainly due to coating asymmetries between CHARA Array telescopes (particularly overcoated silver versus aluminum) that were present in 2010 but removed in 2011. Attempts were made to search for polarization effects that would cause these residuals, but no conclusive evidence was found. Visibilities were, however, never measured in full Stokes parameters in a configuration that showed these residuals.

Because the 2011 data show none of the sinusoidal residuals, our final $\theta_{\mathrm{LD}}$ incorporates the data from 2011 only. This yielded an angular diameter of $0.341 \pm 0.008$ mas. When the data from all nights and using all calibrators are fit together, $\theta_{\mathrm{LD}}$ is 

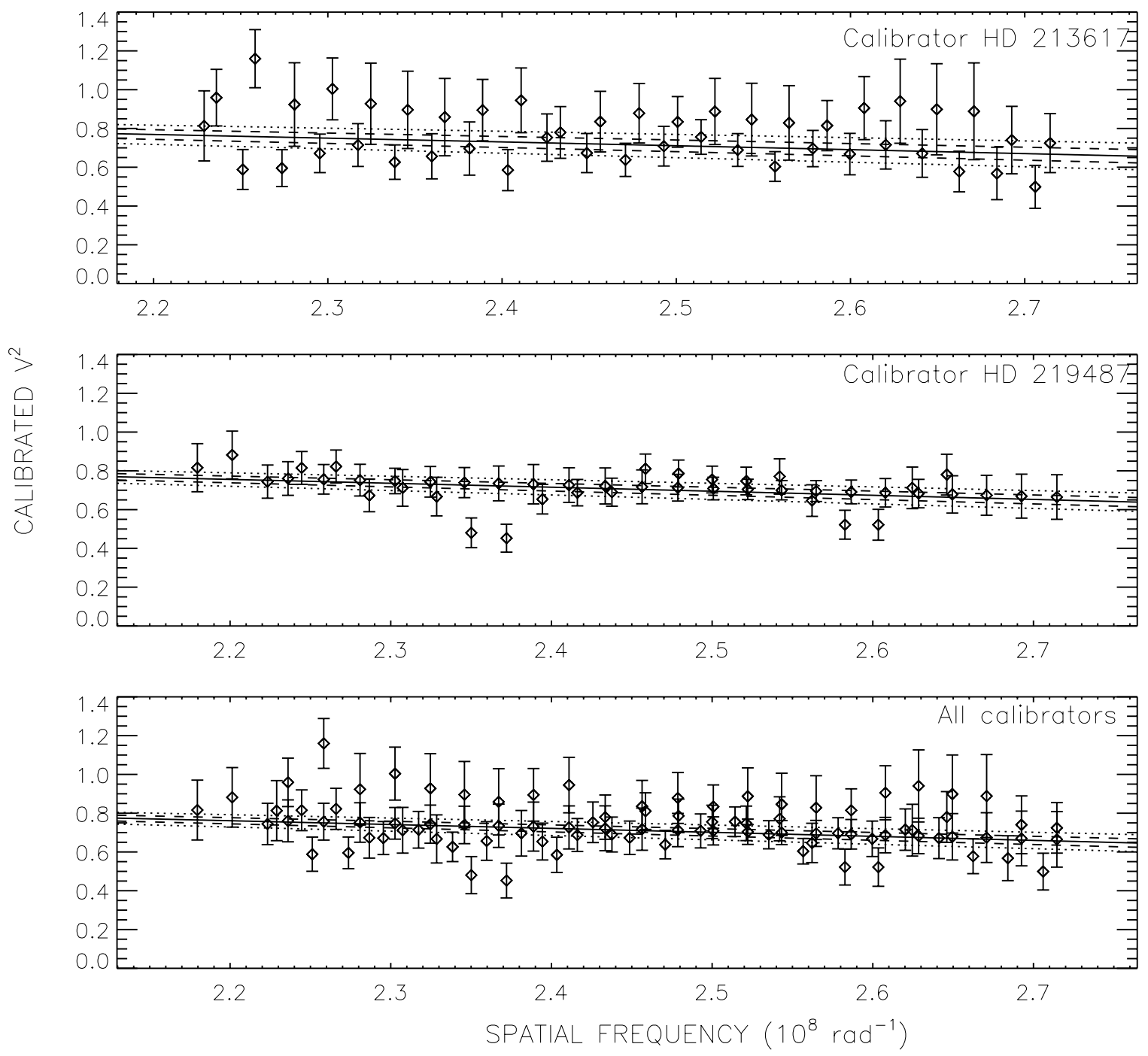

Figure 3. $\theta_{\mathrm{LD}}$ fit for HR 8799 on 2010 September 8 . The symbols are the same as in Figure 1.

$0.347 \pm 0.007$ mas, which is within the uncertainty of the adopted value.

The uncertainty for $\theta_{\mathrm{LD}}$ was calculated as described in the supplemental material from Derekas et al. (2011): for each one of 10,000 Monte Carlo simulated Gaussian distributions, we calibrated the instrumental visibilities and fit an angular diameter to the calibrated data using a least-squares minimization. We accounted for random errors by adding random numbers generated from the empirical covariance matrix scaled by the reduced $\chi^{2}$ of the original fit, and then repeated the procedure. The final uncertainty was the resulting standard deviation of the total distribution.

We observed HR 8799 with multiple calibrator stars every night except one to check on the behavior of the calibrators themselves. For example, when three calibrator stars were used, we used calibrator 1 to determine the angular diameters of calibrator 2 and calibrator 3 to make sure the stars were reliable. The calibrator HD 214698 shows the largest scatter, which is expected because it is the smallest calibrator of the three. There will naturally be more uncertainty when measuring its calibrated visibilities using the other two larger stars. The scatter seen in the night-to-night measurements is expected because the stars are small and very nearly unresolved.

\subsection{Stellar Radius, Luminosity, and Effective Temperature}

At a distance of $39.4 \pm 1.1$ pc (van Leeuwen 2007), HR 8799's $\theta_{\mathrm{LD}}$ of $0.342 \pm 0.008$ mas corresponds to a stellar radius $^{9}$ of $1.44 \pm 0.06 R_{\odot}$, corresponding to a precision of $4 \%$. We note that this radius is $8 \%$ larger than the radius inferred from photometric and temperature considerations in Gray \& Kaye (1999) and subsequently adopted in recent asteroseismic analyses Moya et al. (e.g., 2010a, 2010b); only 1\% of this discrepancy can be attributed to the pre-revised HIPPARCOS distance used in the calculation of Gray \& Kaye (1999).

In order to determine the luminosity $(L)$ and $T_{\text {eff }}$ of HR 8799 , we first constructed its photometric energy distribution (PED) from the averages (when multiple measurements were available) of Johnson $U B V$ magnitudes, Strömgren uvby magnitudes, and Two Micron All Sky Survey (2MASS) JHK magnitudes, as published in Crawford et al. (1966), Breger (1968), Eggen (1968), Mermilliod (1986), Schuster \& Nissen (1986), Mendoza et al. (1990), Hauck \& Mermilliod (1998), Gezari et al. (1999), Høg et al. (2000), Cutri et al. (2003), and Olsen (1983, 1993, 1994). Table 5 summarizes the adopted magnitudes; the assigned uncertainties for the 2MASS infrared measurements

\footnotetext{
9 We define 1 solar radius to be $6.960 \times 10^{10} \mathrm{~cm}$, consistent with an average of previous measurements (Emilio et al. 2012).
} 

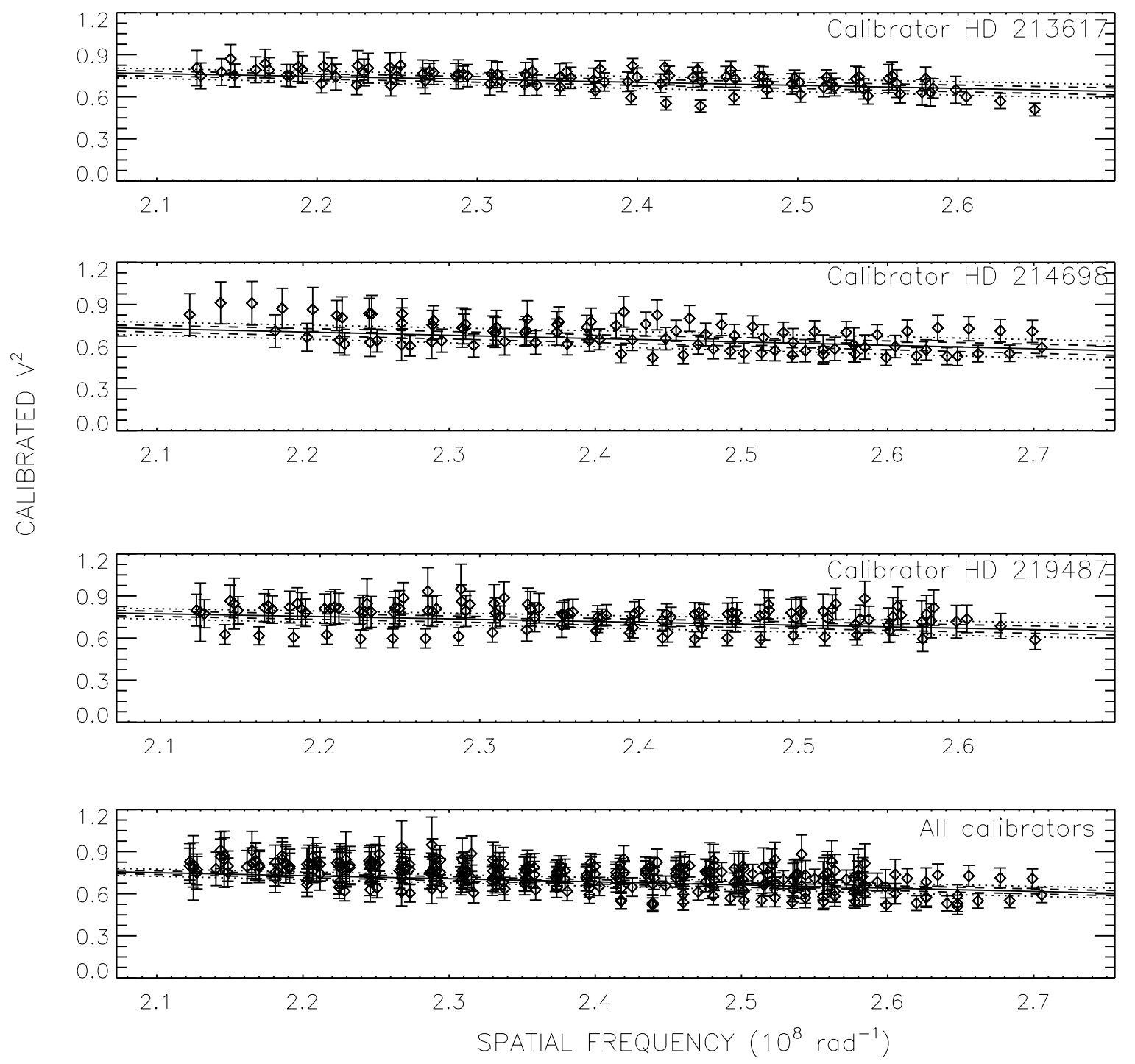

Figure 4. $\theta_{\mathrm{LD}}$ fit for HR 8799 on 2011 October 20. The symbols are the same as in Figure 1.

are as reported, and for optical measurements are standard deviations of multiple measurements. The assigned uncertainties of the optical measurements therefore account for the low amplitude optical variability $( \pm 0.02 \mathrm{mag})$ observed for this variable star (Zerbi et al. 1999).

The bolometric flux $\left(F_{\mathrm{BOL}}\right)$ of HR 8799 was determined by finding the best-fit (via $\chi^{2}$ minimization) stellar spectral template from the flux-calibrated stellar spectral atlas of Pickles (1998). This best PED fit allows for extinction, using the wavelength-dependent reddening relations of Cardelli et al. (1989). The best fit was found using an F0 V template with an assigned temperature of $7211 \pm 90 \mathrm{~K}$, an extinction of $A_{\mathrm{V}}=$ $0.00 \pm 0.01 \mathrm{mag}$, and an $F_{\mathrm{BOL}}$ of $1.043 \pm 0.012 \times$ $10^{-7} \mathrm{erg} \mathrm{s}^{-1} \mathrm{~cm}^{-2}$ (a $1.1 \%$ precision). Figure 11 shows the best fit and the results are listed in Table 6 . To check for possible systematic biases in our adopted prescription, we also fit the PED using synthetic Kurucz spectral models, ${ }^{10}$ assuming no extinction. The resulting $F_{\mathrm{BOL}}$ estimate is consistent to within the $1.1 \%$ error reported above, further validating our technique and measured $F_{\mathrm{BOL}}$.

\footnotetext{
10 Available to download at http://kurucz.cfa.harvard.edu
}

The bolometric flux was then combined with the distance to HR 8799 to estimate its luminosity ( $L=4 \pi \mathrm{d}^{2} F_{\mathrm{BOL}}$ ), which yielded a value of $5.05 \pm 0.29 L_{\odot}$. The uncertainty in the luminosity $(6 \%)$ is predominantly set by the uncertainty in the distance. The $F_{\mathrm{BOL}}$ was also combined with the star's $\theta_{\mathrm{LD}}$ to determine its effective temperature by inverting the relation,

$$
F_{\mathrm{BOL}}=\frac{1}{4} \theta_{\mathrm{LD}}^{2} \sigma T_{\mathrm{eff}}^{4},
$$

where $\sigma$ is the Stefan-Boltzmann constant. This produces an effective temperature of $7203 \pm 87 \mathrm{~K}$, determined to a precision of $1 \%$. Because $\mu_{\mathrm{LD}}$ is chosen using a given $T_{\text {eff }}$, we used our new $T_{\text {eff }}$ value to select $\mu_{\mathrm{LD}}$ and iterated. $\mu_{\mathrm{LD}}$ increased by only 0.01 to $0.49 \pm 0.02, \theta_{\mathrm{LD}}$ increased by 0.001 mas to $0.342 \pm$ 0.008 mas, and $T_{\text {eff }}$ decreased by $10 \mathrm{~K}$ to $7193 \mathrm{~K}$. The very slight change in $\theta_{\mathrm{LD}}$ did not affect the radius calculation at all.

We note that this $T_{\text {eff }}$ is nearly identical to the assigned temperature of the best-fit stellar templates used to calculate $F_{\mathrm{BOL}}$. However, our interferometric $T_{\text {eff }}$ is significantly less model dependent, and it is important to verify the accuracy of the $T_{\text {eff }}$ predicted by the PED. Moreover, our measured $T_{\text {eff }}$ is also consistent with the detailed spectral-type classification by Gray \& Kaye (1999) of kA5 hF0 mA5 v $\lambda$ Boo, following 

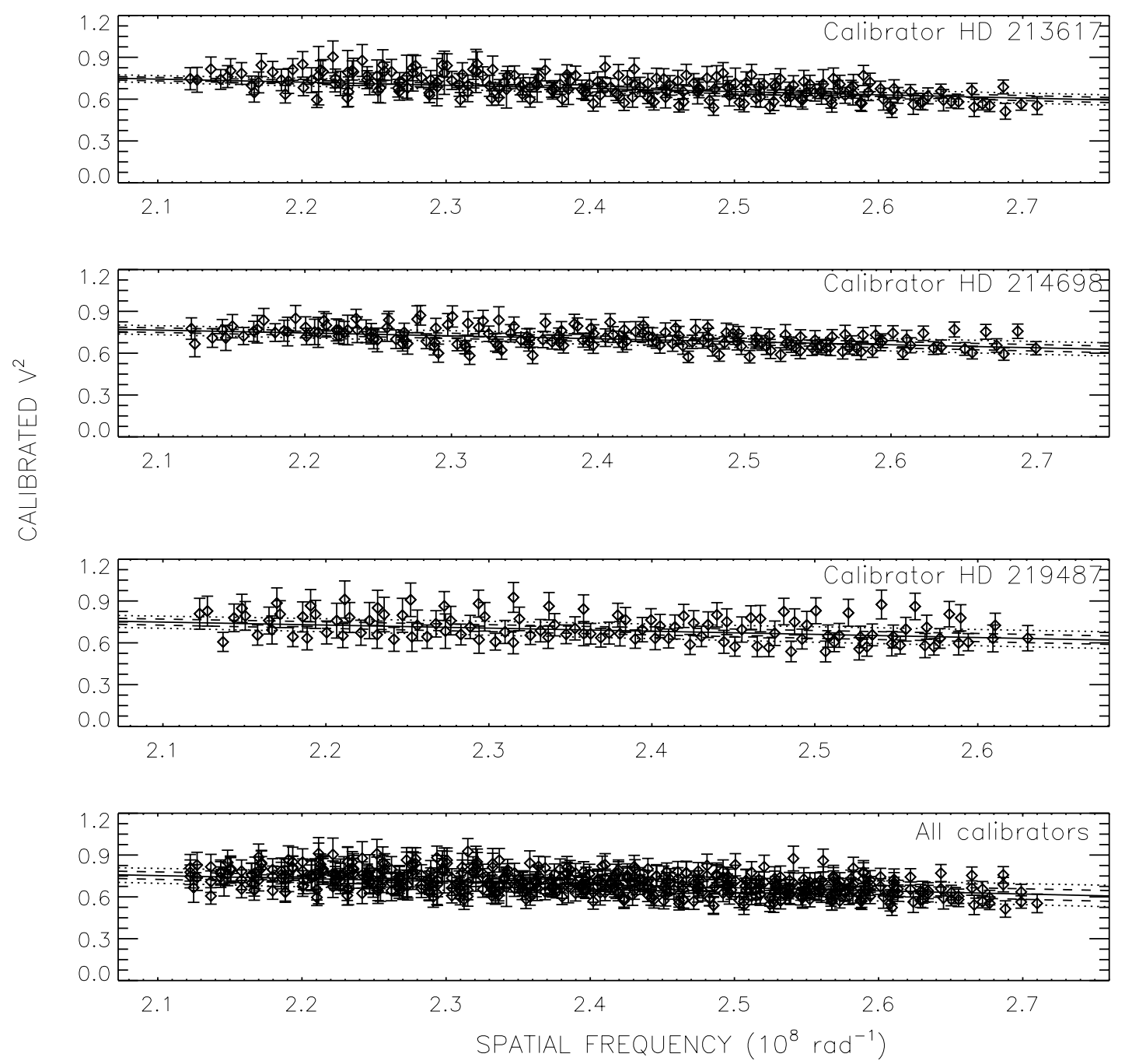

Figure 5. $\theta_{\mathrm{LD}}$ fit for HR 8799 on 2011 October 21 . The symbols are the same as in Figure 1.

the spectral-type temperature scale for dwarf stars assembled in Kraus \& Hillenbrand (2007). The hydrogen lines are a better tracer of the stellar $T_{\text {eff }}(\mathrm{hF0} ; 7200 \mathrm{~K})$; the metal lines suggest a higher $T_{\text {eff }}(\mathrm{mA} 5 ; 8200 \mathrm{~K})$ only because its atmosphere is slightly metal depleted.

A potential bias in the size measurement of any A-type star is oblateness caused by rapid rotation. Royer et al. (2007) measured a $v \sin i$ of $49 \mathrm{~km} \mathrm{~s}^{-1}$ and assuming the planets orbit in the same plane as stellar rotation, the actual rotation velocity will increase from 49 to $104 \mathrm{~km} \mathrm{~s}^{-1}$ when the $28^{\circ}$ inclination is taken into account (Soummer et al. 2011). For an A5 V star with an approximate mass of $2.1 M_{\odot}$ (Cox 2000), our measured radius of $1.44 R_{\odot}$, and the relation between $M$, oblateness, and $v \sin i$ described in van Belle et al. (2006), the predicted oblateness of HR 8799 is only $\sim 2 \%$ and thus within the $1 \sigma$ errors in $\theta_{\mathrm{LD}}$. Using the F0 V spectral type, which is a closer fit to our measured $T_{\text {eff }}$, the estimated mass is $1.6 M_{\odot}$ and the predicted oblateness is $2.6 \%$, still a small effect.

\section{DISCUSSION}

\subsection{Habitable Real-estate of HR 8799}

HR 8799 is currently the only directly imaged multiple planet system and there may be smaller, more Earth-like planets orbiting the star or even moons orbiting the imaged planets that have not yet been detected. This would present the possibility of life if the planets were in the habitable zone (HZ) of the star, so we used our new precise measurements to improve the estimate of the system's HZ. We used the following equations from Jones et al. (2006):

$S_{b, i}\left(T_{\text {eff }}\right)=\left(4.190 \times 10^{-8} T_{\text {eff }}^{2}\right)-\left(2.139 \times 10^{-4} T_{\text {eff }}\right)+1.296$

and

$$
\begin{aligned}
S_{b, o}\left(T_{\text {eff }}\right)= & \left(6.190 \times 10^{-9} T_{\text {eff }}^{2}\right)-\left(1.319 \times 10^{-5} T_{\text {eff }}\right) \\
& +0.2341
\end{aligned}
$$

where $S_{b, i}\left(T_{\text {eff }}\right)$ and $S_{b, o}\left(T_{\text {eff }}\right)$ are the critical fluxes at the inner and outer boundaries in units of the solar constant. The inner and outer physical boundaries $r_{i, o}$ in AU were then calculated using

$$
r_{i}=\sqrt{\frac{L / L_{\odot}}{S_{b, i}\left(T_{\mathrm{eff}}\right)}} \quad \text { and } \quad r_{o}=\sqrt{\frac{L / L_{\odot}}{S_{b, o}\left(T_{\mathrm{eff}}\right)}}
$$

These equations assume that the $\mathrm{HZ}$ is dependent on distance only and do not take other effects, such as tidal heating, into account. The inner boundary is the limit where a runaway 

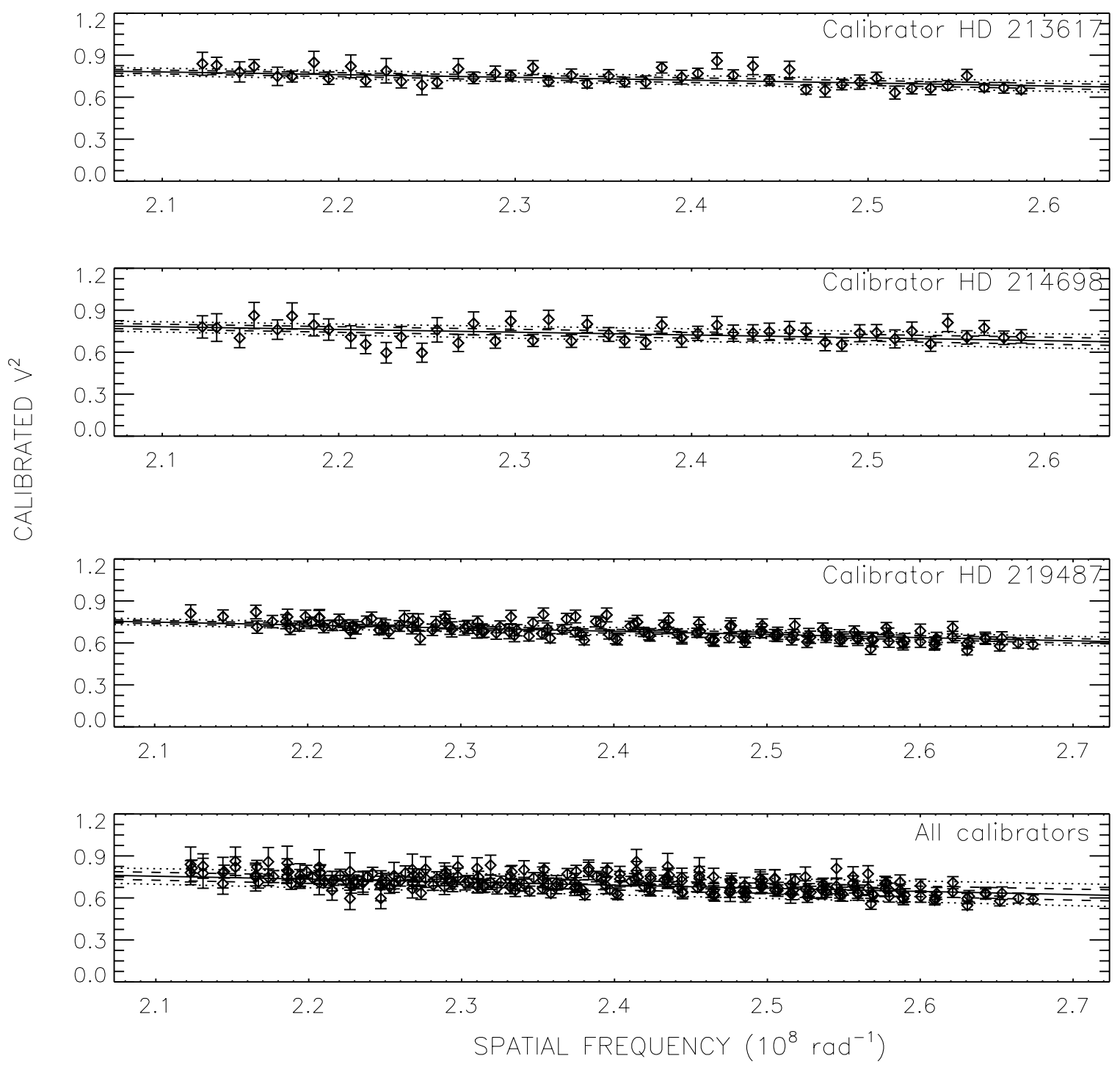

Figure 6. $\theta_{\mathrm{LD}}$ fit for HR 8799 on 2011 October 22. The symbols are the same as in Figure 1.

greenhouse effect would evaporate any surface water while the outer boundary is the limit where a cloudless atmosphere could maintain a surface temperature of $273 \mathrm{~K}$. Jones et al. (2006) note that these equations produce a conservatively small $\mathrm{HZ}$ and the actual HZ may be wider.

We obtained HZ boundaries of 1.62 AU and 3.32 AU. HR 8799's planets have semimajor axes of 14.5-68 AU (Marois et al. $2008,2010)$. There is no chance the planets orbit anywhere near the $\mathrm{HZ}$ unless they are highly eccentric, which is a configuration more likely to be unstable.

\subsection{Effective Abundance of the $\lambda$ Boo Stars and HR 8799}

The accurately determined stellar properties of HR 8799 also allow for detailed comparisons with stellar evolutionary models from which a mass and age can be inferred. However, correctly interpreting masses and especially ages depends critically upon knowing the internal abundances of this peculiar abundance star. As noted in Section 1, the atmospheric abundances of Fe-peak elements are distinctly subsolar $([\mathrm{Fe} / \mathrm{H}] \sim-0.4$ dex $)$ while the abundances of $\mathrm{C}, \mathrm{N}, \mathrm{O}$, and $\mathrm{S}$ are essentially solar. Because these surface abundances may not directly trace the internal abundances, it is unclear which, if any, of the available uniformly scaled abundance models to adopt for these comparisons; we subsequently refer to the abundance of the uniformly scaled model that predicts properties most consistent with observational constraints as the effective abundance.

As an example of the effect on the inferred ages, comparisons of HR 8799 with models that assume subsolar abundances throughout yield ages of $1.05 \pm 0.26 \mathrm{Gyr}$ for $[\mathrm{Fe} / \mathrm{H}]=-0.27$ or $1.71 \pm 0.18 \mathrm{Gyr}$ for $[\mathrm{Fe} / \mathrm{H}]=-0.43$, while those that assume near-solar abundances $([\mathrm{Fe} / \mathrm{H}]=+0.05)$ yield an age of $<0.1 \mathrm{Gyr}$; the specific physical assumptions used in these models are described in Section 4.3. Obviously, the polyabundic atmospheres of $\lambda$ Boo stars and their uncertain internal abundance compromises the validity of these comparisons and the inferred values. Nevertheless, we present the available observational evidence for constraining the metallicity of $\lambda$ Boo stars, and HR 8799 in particular, and suggest the effective abundance of HR 8799, and perhaps all $\lambda$ Boo stars, is near solar.

The observationally favored theory to explain the $\lambda$ Boo phenomenon, originally proposed by Venn \& Lambert (1990), is that the atmospheres have been polluted by the accretion of Fe-peak depleted gas. Depletion is believed to occur because of grain formation; a similar depletion of Fe-like elements has been observed in interstellar clouds (Morton 1974). The grains that themselves accrete Fe-elements are consequently inhibited from accreting on to the star because of the stronger radiation 


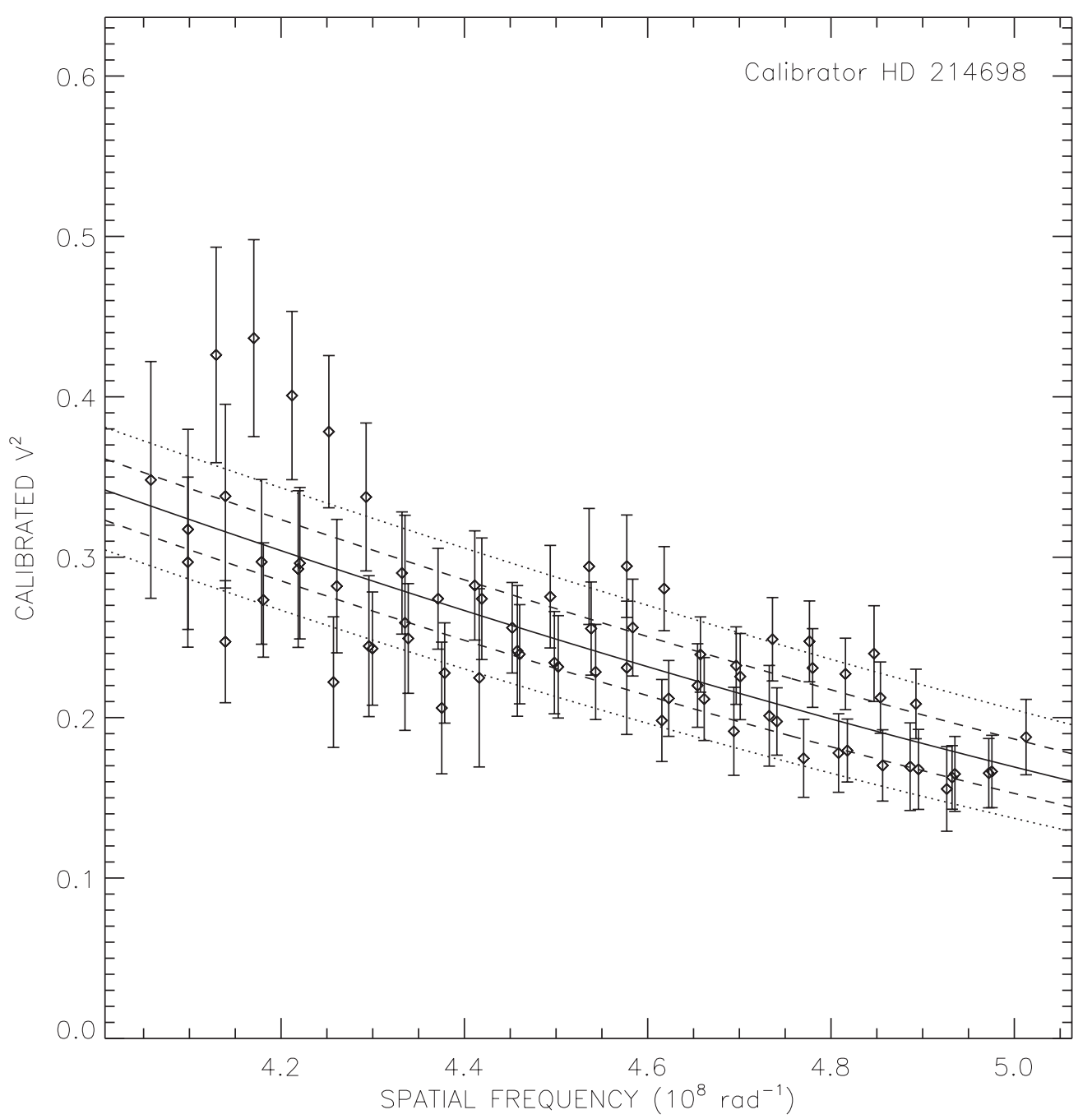

Figure 7. $\theta_{\text {LD }}$ fit for HR 8799 on 2011 September 30. The symbols are the same as in Figure 1.

pressure they experience. It is not clear why this accretion onto the grains occurs, or where the accreting material comes from. It may be interstellar, but in many cases appears to be circumstellar. As noted by Gray \& Corbally (2002), all four $\lambda$ Boo stars within 40 pc (including HR 8799) exhibit an infrared excess that is interpreted as the presence of circumstellar dust, while only $\sim 18 \%$ of non- $\lambda$ Boo A-type stars exhibit such excesses. The apparent depletion of these elements requires only relatively low accretion rates $\left(10^{-13} M_{\odot} \mathrm{yr}^{-1}\right)$, because of the shallow convective zones in A-type stars (Charbonneau 1991). Accretion at these rates will quickly establish abundance anomalies within a few Myr, but these anomalies will likewise disappear in as little as $1 \mathrm{Myr}$ once the accretion has terminated (Turcotte \& Charbonneau 1993); the interesting implication is that all $\lambda$ Boo stars are either currently accreting, or have only recently terminated their accretion. If this favored theory is correct, it suggests that the depleted Fe-peak abundances do not represent the internal abundances of these stars. Given this and the typical limiting main-sequence lifetime of $\sim 2$ Gyr for these intermediate mass Population I stars, ${ }^{11}$ one would expect their internal abundance to be on average close to solar.

We note that an alternative mechanism to explain the $\lambda$ Boo phenomenon proposed by Michaud \& Charland (1986) suggests

\footnotetext{
11 Studies have shown that stars currently forming in open clusters are
} generally metal-rich (e.g., Santos et al. 2009). that the depletion of Fe-peak elements is a result of diffusion and mass loss. In this case, $\lambda$ Boo stars are much closer to the end of their main-sequence lifetimes. To be effective, the star would need to be losing mass at a rate of the order of $10^{-13} M_{\odot} \mathrm{yr}^{-1}$ for a few times $10^{8} \mathrm{yr}$ and thus implies that the $\lambda$ Boo phenomenon be restricted to the end of the main-sequence evolutionary phase for A stars. This predicted timescale is difficult to reconcile with the discovery of very young $\lambda$ Boo stars in the Orion OB1 associations (age $<10 \mathrm{Myr}$ ), and the lack of any $\lambda$ Boo stars in intermediate age open clusters (Gray $\&$ Corbally 2002). Evidence such as the higher fraction of these stars with circumstellar debris disks, noted above, instead suggest that the $\lambda$ Boo phenomenon is more common among young A stars. If true, this would strengthen the case that the internal abundances of these Population I stars, at least on average, should be close to solar. In particular, we note that the existence of $\lambda$ Boo stars in the Orion star-forming region implies that their primordial abundances are solar, consistent with other stars in this cluster (Nieva \& Przybilla 2012). If the depletion of Fe-like elements is restricted to only the outer atmospheres, then one can conclude that in these cases the internal abundances should likewise be solar.

As described in Marois et al. (2008), the space motions of HR 8799 are consistent with those of young stars in the solar neighborhood. We investigated this further by comparing the 

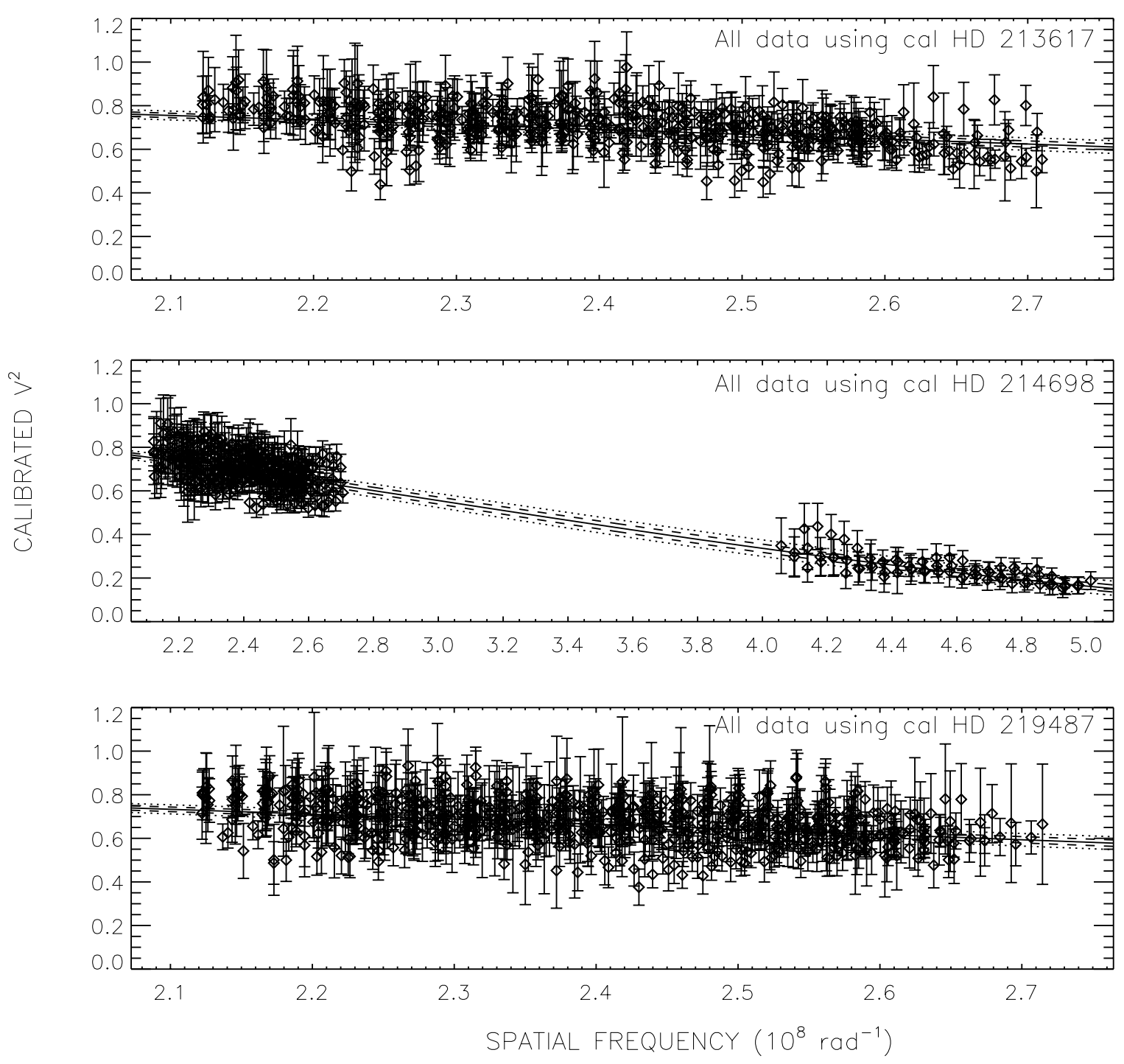

Figure 8. $\theta_{\mathrm{LD}}$ fit for HR 8799 by calibrator. The symbols are the same as in Figure 1.

$U V W$ space motions of HR 8799 to those of nearby moving groups assembled in Torres et al. (2008); kinematic association with a moving group would not only help constrain the age of the system but also the primordial abundance of the star. We calculated $U V W$ space motions using HR 8799's HIPPARCOS distance and parallax (van Leeuwen 2007), and a radial velocity of $-12.6 \pm 1.3 \mathrm{~km} \mathrm{~s}^{-1}$ from Barbier-Brossat \& Figon (2000). This yielded velocities of $U=-12.24 \pm 0.37 \mathrm{~km} \mathrm{~s}^{-1}, V=$ $-21.22 \pm 1.10 \mathrm{~km} \mathrm{~s}^{-1}$, and $W=-7.15 \pm 0.86 \mathrm{~km} \mathrm{~s}^{-1}$; these are all within $\sim 1 \mathrm{~km} \mathrm{~s}^{-1}$ of the values reported in Marois et al. (2008). Figure 12 illustrates the space motion of HR 8799 relative to that of several kinematically similar moving groups after adopting moving group velocities and uncertainties from Torres et al. (2008). Although the space motion of HR 8799 is not consistent to within $1 \sigma$ of any of these groups, it is consistent to within $3 \sigma$ of two of these groups: Columba $(1.2 \sigma)$, and $\epsilon$ Cha $(2.2 \sigma)$. We thus confirm the report by Marois et al. (2008) of the potentially youthful kinematics of HR 8799, which favors but cannot confirm an age less than $\lesssim 1 \mathrm{Gyr}$, and likely a solar abundance consistent with many of these stars.

As described in Section 1, there are two other aspects of HR 8799 that argue for a young age for the star and system. The strong infrared excess of HR 8799 statistically favors an age less than 500 Myr (Gáspár et al. 2009), despite the challenge raised by Moya et al. (2010a). An even stronger youthful age restriction is imposed by the dynamical considerations of MoroMartín et al. (2010). Although their conclusions require use of the predictions of poorly constrained planetary evolutionary models, even considerable uncertainties in the mass estimates require the age of the system to be less than a few $100 \mathrm{Myr}$, otherwise the planets would be too massive to be orbitally stable. The ages much less than 1 Gyr for HR 8799 can only be reconciled if the adopted effective abundances are near solar.

Recently, Moya et al. (2010b) used the $\gamma$ Dor-type pulsations of HR 8799 to asteroseismically constrain the star's internal metallicity. They find a best-fit internal metallicity of $-0.32 \pm$ 0.1 , assuming that the rotation axis of the star, which affects the rotation speed of the star, is somewhat highly inclined relative to our line of sight $\left(50^{\circ}\right)$. This high inclination is inconsistent with the more face-on orientation of HR 8799's disk (Su et al. 2009) and the apparent orbital plane of its companions (Lafrenière et al. 2009), although these need not be coplanar. The asteroseismology analysis is compromised by the use of an assumed radius that is too small by $8 \%$ and a temperature that is too hot by $3 \%$ relative to the precisely determined values in this study. The measured $R$ and $T_{\text {eff }}$ presented here will 

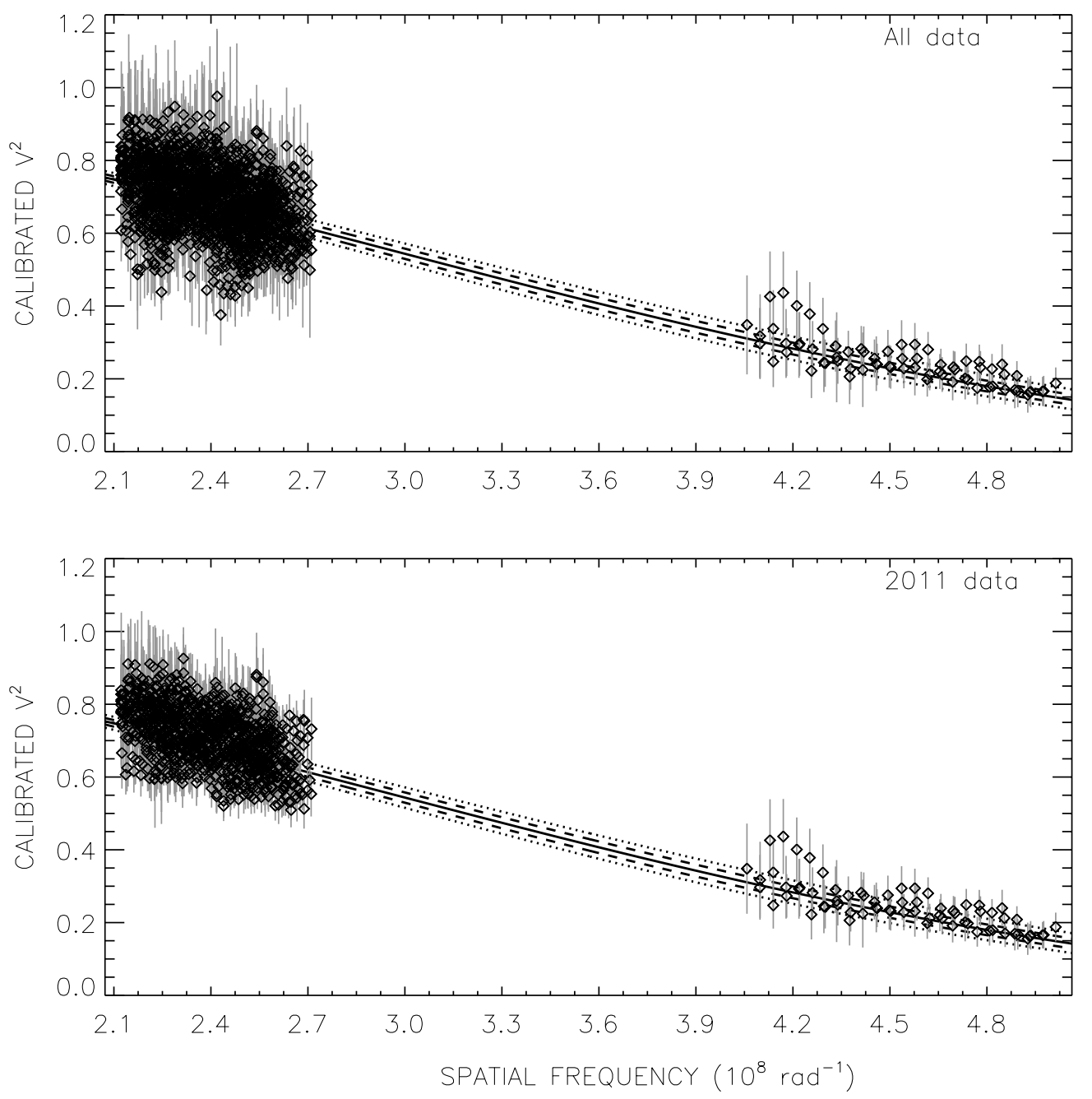

Figure 9. $\theta_{\mathrm{LD}}$ fit for HR 8799 using all calibrators. The symbols are the same as in Figure 1.

be valuable to constrain the model parameter space in future asteroseismic modeling efforts, which will possibly constrain the internal metallicity.

Moya et al. (2010b) do find possible-though less likely-solutions with internal abundances close to solar $([\mathrm{Fe} / \mathrm{H}]=-0.12)$ for closer to pole-on orientations; this is the most metal-rich metallicity reported in their study. Overall, we find the results of this metallicity analysis to be inconclusive primarily because of the large uncertainty in the inclination of the star's rotation axis, which Moya et al. acknowledge is the limiting factor in their analysis. If anything, the result that they find some acceptable solutions with near-solar metallicities and young ages corroborated previous asteroseismology studies of $\lambda$ Boo stars. Using densities inferred from stellar pulsations, Paunzen et al. (1998) found the location of most $\lambda$ Boo stars showing $\delta$ Scuti pulsations on a plot of average density versus period to be consistent with the positions of $\delta$ Scuti stars with solar abundances. They interpret this as evidence that the low Fepeak abundances are restricted to the surface of $\lambda$ Boo stars and do not represent the state of the interior composition. Although the variations in HR 8799 are compatible with $\gamma$ Dor pulsations, the large number of discovered hybrid $\delta \mathrm{Scu}-\gamma$ Dor pulsators (e.g., Rowe et al. 2006; Grigahcène et al. 2010) suggests that this argument could be applicable for all $\lambda$ Boo stars.

In summary, based on the observational evidence supporting the accretion of clean gas theory explaining the abundance pattern of $\lambda$ Boo stars, the existence of at least some $\lambda$ Boo stars in an OB Association, the evidence of youth for many $\lambda$ Boo stars (and especially HR 8799), the restriction of ages less than $\sim 2$ Gyr for Population I stars, and the available asteroseismic constraints on internal metallicity, we conclude that the most appropriate effective abundances for $\mathrm{HR}$ 8799 , and quite possibly all $\lambda$ Boo stars, is near solar. Until more sophisticated evolutionary models that account for the polyabundic atmospheres and possibly interiors of these stars are developed, we are hopeful that $\lambda$ Boo stars in clusters or with lower mass companions will be discovered, which will enable improvements in both assigning effective abundances and tests of the validity of using uniformly scaled abundances.

\subsection{Mass and Age of HR 8799 and Implications for Its Companions}

Following the above arguments, we estimated the mass and age of HR 8799 using the stellar evolutionary models of the Yonsei-Yale group ( $\mathrm{Y}^{2}$; Yi et al. 2001), updated by Demarque et al. (2004) to account for convective core overshoot. The models handle convection using mixing length prescription, adopting a mixing length of 1.7432 times the pressure scale height that is set by comparisons with a solar model. The model uses the solar abundances of Grevesse et al. (1996) and OPAL radiative opacities (Rogers \& Iglesias 1995; Iglesias \& Rogers 


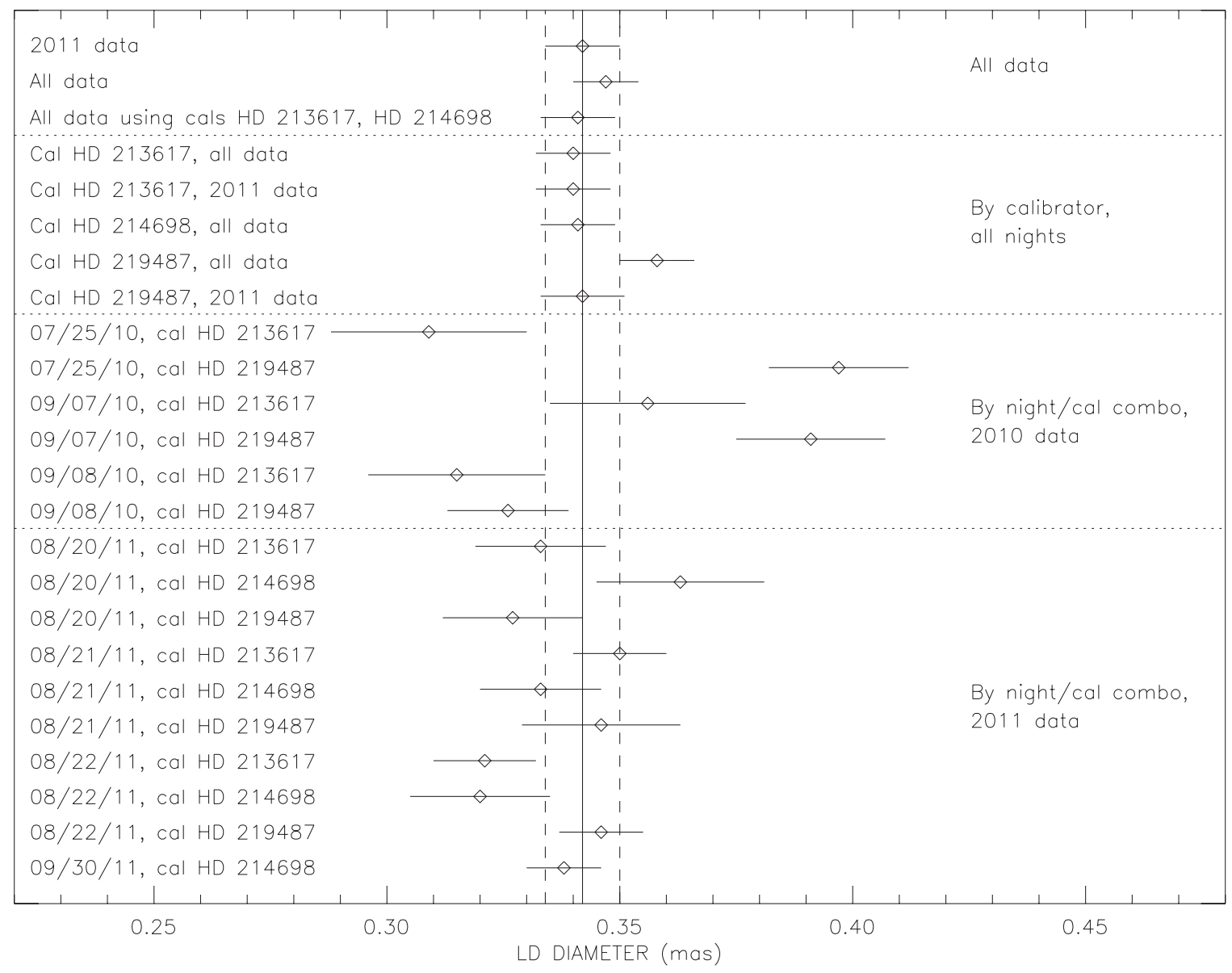

Figure 10. Comparison of $\theta_{\mathrm{LD}}$ fits by individual night and calibrator. The vertical solid line represents the final adopted $\theta_{\mathrm{LD}}(0.342 \pm 0.008 \mathrm{mas}$, the top line $)$ and the vertical dashed lines are the errors in that fit. Table 4 lists the numerical values. Note the reduced scatter in the 2011 data vs. the 2010 data.

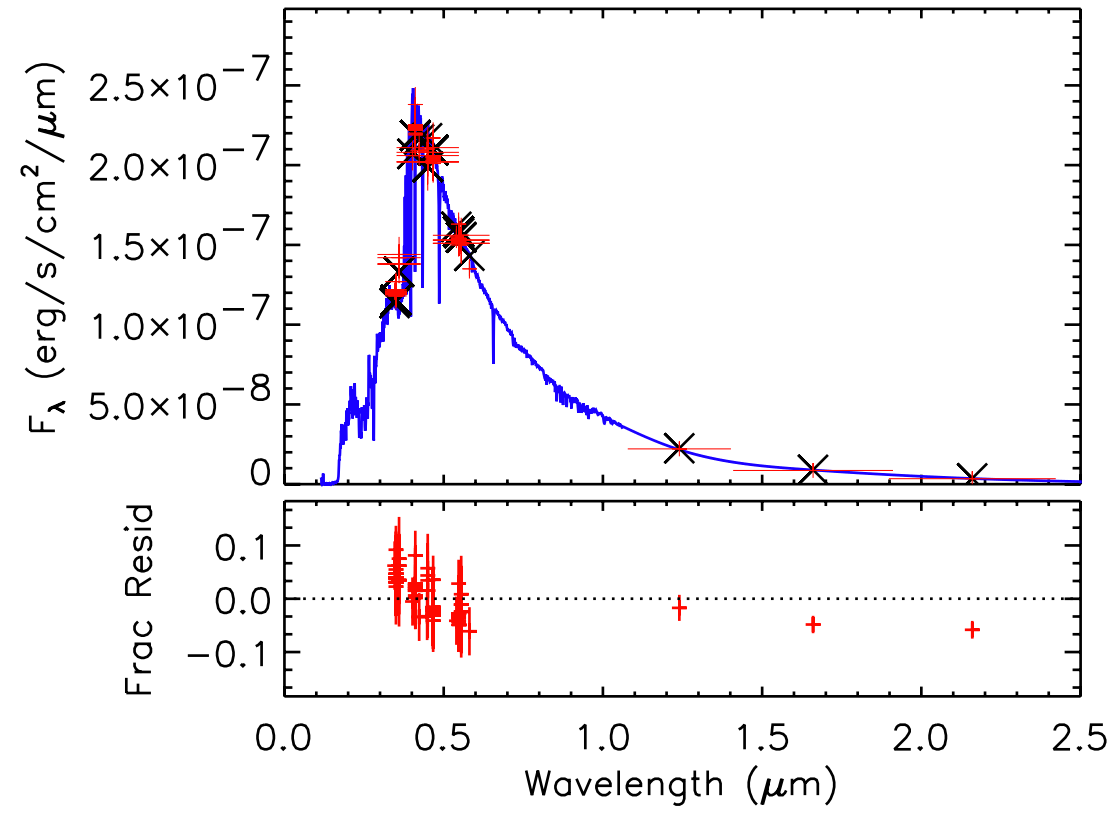

Figure 11. HR 8799 PED fit. Upper panel: the solid-line spectrum is an F0 V spectral template from Pickles (1998). The crosses indicate photometry values from the literature. The horizontal bars represent bandwidths of the filters used. The X-shaped symbols show the flux value of the spectral template integrated over the filter transmission. Lower panel: the crosses are the residuals around the fit in fractional flux units of photometric uncertainty. For more details, see Section 4.

(A color version of this figure is available in the online journal.)

1996) for the interior and the Alexander \& Ferguson (1994) opacities for the cooler, outer regions of the star. Additional information regarding the input physics assumed in these models can be found in Yi et al. (2001) and Demarque et al. (2004).
However, since a set solar metallicity models is not provided, we followed the recommendation of the modelers themselves and generated a set of solar metallicity models by linearly interpolating isochrones and mass tracks between nearest the 

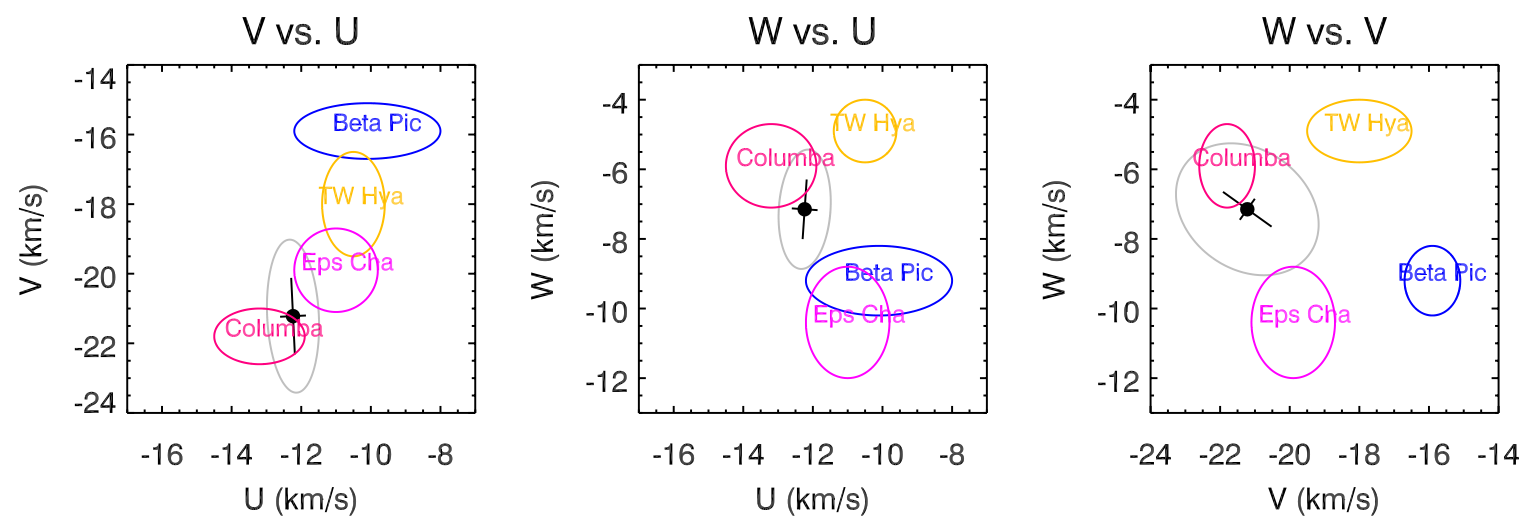

Figure 12. $U V W$ space velocities of HR 8799 (black point) with $1 \sigma$ (error bars) and $2 \sigma$ (gray ellipse) errors. Also plotted are the $U V W$ space velocities and $1 \sigma$ errors (as ellipses) of four young stellar associations with similar kinematics, as taken from Torres et al. (2008). HR 8799 matches the space velocity of the roughly 30 Myr old Columba association to $1.2 \sigma$.

(A color version of this figure is available in the online journal.)

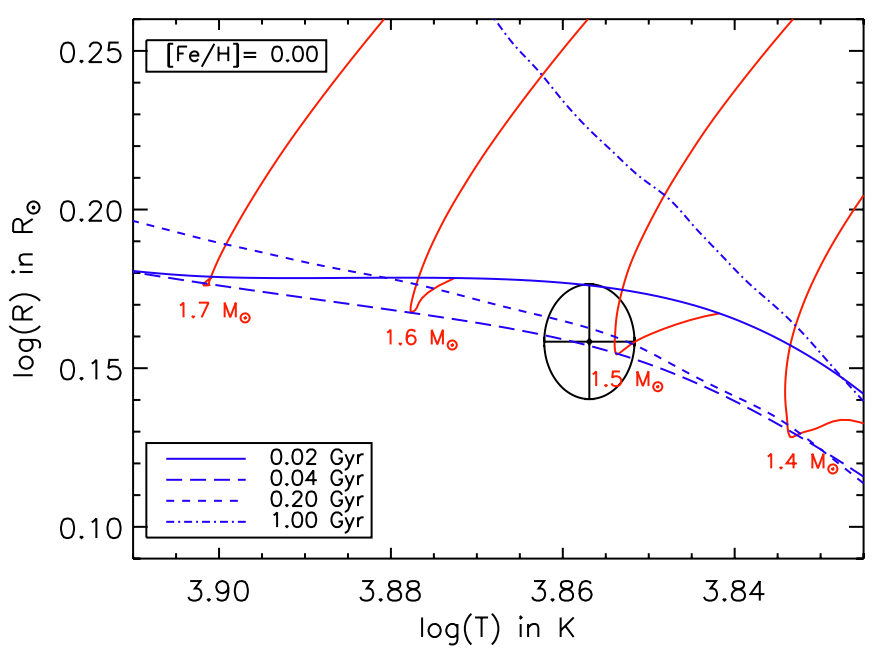

Figure 13. Radius and temperature of HR 8799 plotted along with isochrones (blue lines) and mass tracks (solid red lines) from Demarque et al. (2004), with solar abundances. Note that the mass tracks predict that these stars are still gravitationally settling at $0.02 \mathrm{Gyr}$, reach their smallest size at $0.04 \mathrm{Gyr}$, and expand in size thereafter.

(A color version of this figure is available in the online journal.)

metallicity models of $[\mathrm{Fe} / \mathrm{H}]=-0.27 \mathrm{dex}$ and $[\mathrm{Fe} / \mathrm{H}]=+0.05$ dex (models x74z01 and x71z04 models, respectively). The solar metallicity models are illustrated in Figure 13.

At HR 8799's temperature, stars are predicted to have fully contracted to their smallest size, or the zero-age main sequence (ZAMS) at $40 \mathrm{Myr}$, and then begin expanding due to stellar evolutionary effects at a much slower rate afterward. As such, HR 8799 is either contracting onto the ZAMS or expanding from it, and we use the $40 \mathrm{Myr}$ isochrone as the upper limit for the age in the pre-main-sequence scenario and as the lower limit for the age in the post-main-sequence scenario. More specifically, we find that if HR 8799 is contracting onto the ZAMS, it has an age of $33_{-13.2}^{+7} \mathrm{Myr}$ and a mass of $1.516_{-0.024}^{+0.038} M_{\odot}$. If HR 8799 is expanding from the ZAMS, we find it to have an age of $90_{-50}^{+381} \mathrm{Myr}$ and a mass of $1.513_{-0.024}^{+0.023} M_{\odot}$. These masses and ages are found by interpolating between the solar metallicity models described above. The masses are consistent with the mass used in Marois et al. (2008), which was $1.5 \pm 0.3 M_{\odot}$. We remind the reader that these quoted errors on the star's mass and age are statistical and therefore do not take into account uncertainties in the metallicity and/or models themselves.

With an age of $\lesssim 0.1 \mathrm{Gyr}$, the companions that HR 8799 harbors are more likely to have planetary masses. As explained in Marois et al. (2008), planetary mass objects could only be as bright as these observed companions are if they are young. That inferred age, and thus companion masses, depends critically upon adopted evolutionary model abundance. While near solar seems likely, even slightly subsolar abundances can give uncertainties in age that extend to above $\sim 500 \mathrm{Myr}$, increasing the inferred companion masses by at least a factor of two.

\section{SUMMARY}

We measured the angular diameter of HR 8799 using the CHARA Array interferometer and used our new value of $0.342 \pm 0.008$ mas to calculate the star's physical radius $\left(1.44 \pm 0.06 R_{\odot}\right)$, luminosity $\left(5.05 \pm 0.29 L_{\odot}\right)$, and effective temperature $(7193 \pm 87 \mathrm{~K})$ by combining our measurement with information from the literature. We used our $T_{\text {eff }}$ measurement to determine the size of the HZ, which is well inside the orbits of any of the companions detected to date.

Based on a variety of techniques, we concluded that the most appropriate abundances for HR 8799 are close to solar. We combined our $R$ and $T_{\text {eff }}$ values with $\mathrm{Y}^{2}$ isochrones to estimate the star's mass and age in two scenarios: $1.516_{-0.024}^{+0.038} M_{\odot}$ and $33_{-13.2}^{+7}$ Myr if the star is contracting onto the ZAMS or $1.513_{-0.024}^{+0.023} M_{\odot}$ and $90_{-50}^{+381} \mathrm{Myr}$ if it is expanding from it. In either case, this young age implies the imaged companions are planets and not brown dwarfs. The only case in which the companions would be close to brown dwarf mass is if the highest age of $471 \mathrm{Myr}$ was the true case, and even then the masses would be on the exoplanet/brown dwarf cusp.

We thank Gerard van Belle for his insight on the nature of HR 8799's pirouette through space. The CHARA Array is funded by the National Science Foundation through NSF grant AST-0606958 and by Georgia State University through the College of Arts and Sciences, and S.T.R. acknowledges partial support by NASA grant NNH09AK731. This research has made use of the SIMBAD database, operated at CDS, Strasbourg, France. This publication makes use of data products from the Two Micron All Sky Survey, which is a joint project of the 
University of Massachusetts and the Infrared Processing and Analysis Center/California Institute of Technology, funded by the National Aeronautics and Space Administration and the National Science Foundation.

\section{REFERENCES}

Alexander, D. R., \& Ferguson, J. W. 1994, ApJ, 437, 879

Allende Prieto, C., \& Lambert, D. L. 1999, A\&A, 352, 555

Barbier-Brossat, M., \& Figon, P. 2000, A\&AS, 142, 217

Bessell, M. S. 2000, PASP, 112, 961

Breger, M. 1968, AJ, 73, 84

Breger, M. 1979, PASP, 91, 5

Cardelli, J. A., Clayton, G. C., \& Mathis, J. S. 1989, ApJ, 345, 245

Charbonneau, P. 1991, ApJ, 372, L33

Chen, C. H., Sargent, B. A., Bohac, C., et al. 2006, ApJS, 166, 351

Claret, A., \& Bloemen, S. 2011, A\&A, 529, A75

Cox, A. N. 2000, Allen's Astrophysical Quantities (Melville, NY: AIP)

Crawford, D. L., Barnes, J. V., Faure, B. Q., \& Golson, J. C. 1966, AJ, 71, 709

Currie, T., Burrows, A., Itoh, Y., et al. 2011, ApJ, 729, 128

Cutri, R. M., Skurtskie, M. F., van Dyk, S., et al. 2003, The IRSA 2MASS All-Sky Point Source Catalog, NASA/IPAC Infrared Science Archive, http://irsa.ipac.caltech.edu/applications/Gator/

Demarque, P., Woo, J.-H., Kim, Y.-C., \& Yi, S. K. 2004, ApJS, 155, 667

Derekas, A., Kiss, L. L., Borkovits, T., et al. 2011, Science, 332, 216

Doyon, R., Lafrenière, D., Artigau, E., Malo, L., \& Marois, C. 2010, in Proc. Conf. In the Spirit of Lyot: The Direct Detection of Planets and Circumstellar Disks, ed. A. Boccaletti (Paris: LESIA/CNRS), 42

Eggen, O. J. (ed.) 1968, Photography for the Scientist (London: Academic), 37 Emilio, M., Kuhn, J. R., Bush, R. I., \& Scholl, I. F. 2012, ApJ, 750, 135

Fabrycky, D. C., \& Murray-Clay, R. A. 2010, ApJ, 710, 1408

Gáspár, A., Rieke, G. H., Su, K. Y. L., et al. 2009, ApJ, 697, 1578

Gezari, D. Y., Pitts, P. S., \& Schmitz, M. 1999, VizieR Online Data Catalog, 2225,0

Goździewski, K., \& Migaszewski, C. 2009, MNRAS, 397, L16

Gray, R. O., \& Corbally, C. J. 2002, AJ, 124, 989

Gray, R. O., \& Kaye, A. B. 1999, AJ, 118, 2993

Grevesse, N., Noels, A., \& Sauval, A. J. 1996, Cosm. Abundances, 99, 117

Grigahcène, A., Uytterhoeven, K., Antoci, V., et al. 2010, Astron. Nachr., 331, 989

Hanbury Brown, R., Davis, J., Lake, R. J. W., \& Thompson, R. J. 1974, MNRAS, 167,475

Handler, G. 1999, MNRAS, 309, L19

Hauck, B., \& Mermilliod, M. 1998, A\&AS, 129, 431

Høg, E., Fabricius, C., Makarov, V. V., et al. 2000, A\&A, 355, L27

Iglesias, C. A., \& Rogers, F. J. 1996, ApJ, 464, 943

Ireland, M. J., Mérand, A., ten Brummelaar, T. A., et al. 2008, Proc. SPIE, 7013 , 63

Jones, B. W., Sleep, P. N., \& Underwood, D. R. 2006, ApJ, 649, 1010

Kaye, A. B., Handler, G., Krisciunas, K., Poretti, E., \& Zerbi, F. M. 1999, PASP, 111,840

Kervella, P., Thévenin, F., Di Folco, E., \& Ségransan, D. 2004, A\&A, 426, 297

Kraus, A. L., \& Hillenbrand, L. A. 2007, AJ, 134, 2340

Lafrenière, D., Jayawardhana, R., \& van Kerkwijk, M. H. 2010, ApJ, 719, 497

Lafrenière, D., Marois, C., Doyon, R., \& Barman, T. 2009, ApJ, 694, L148

Marley, M. S., Saumon, D., Cushing, M., et al. 2012, ApJ, 754, 135
Marois, C., Macintosh, B., Barman, T., Zuckerman, B., et al. 2008, Science, 322, 1348

Marois, C., Zuckerman, B., Konopacky, Q. M., Macintosh, B., \& Barman, T. 2010, Nature, 468, 1080

Mendoza, E. E., Rolland, A., \& Rodriguez, E. 1990, A\&AS, 84, 29

Mermilliod, J.-C. 1986, Catalogue of Eggen's UBV Data, 0

Mermilliod, J. C. 1991, Catalogue of Homogeneous Means in the UBV System (Lausanne: Institut d'Astronomie, Universite de Lausanne)

Michaud, G., \& Charland, Y. 1986, ApJ, 311, 326

Moór, A., Ábrahám, P., Derekas, A., et al. 2006, ApJ, 644, 525

Moro-Martín, A., Rieke, G. H., \& Su, K. Y. L. 2010, ApJ, 721, L199

Morton, D. C. 1974, ApJ, 193, L35

Moya, A., Amado, P. J., Barrado, D., et al. 2010a, MNRAS, 405, L81

Moya, A., Amado, P. J., Barrado, D., et al. 2010b, MNRAS, 406, 566

Nieva, M.-F., \& Przybilla, N. 2012, A\&A, 539, A143

O’Donnell, J. E. 1994, ApJ, 422, 158

Olsen, E. H. 1983, A\&AS, 54, 55

Olsen, E. H. 1993, A\&AS, 102, 89

Olsen, E. H. 1994, A\&AS, 106, 257

Paunzen, E., Weiss, W. W., Kuschnig, R., et al. 1998, A\&A, 335, 533

Pecaut, M. J., Mamajek, E. E., \& Bubar, E. J. 2012, ApJ, 746, 154

Perryman, M. A. C., Brown, A. G. A., Lebreton, Y., et al. 1998, A\&A, 331, 81

Perryman, M. A. C., \& ESA 1997, The Hipparcos and Tycho Catalogs (SP-1200; Noordwjk: ESA)

Pickles, A. J. 1998, PASP, 110, 863

Rhee, J. H., Song, I., Zuckerman, B., \& McElwain, M. 2007, ApJ, 660, 1556

Reidemeister, M., Krivov, A. V., Schmidt, T. O. B., et al. 2009, A\&A, 503,247

Rogers, F. J., \& Iglesias, C. A. 1995, Highlights Astron., 10, 573

Rowe, J. F., Matthews, J. M., Cameron, C., et al. 2006, Commun. Asteroseismol., 148,34

Royer, F., Zorec, J., \& Gómez, A. E. 2007, A\&A, 463, 671

Sadakane, K. 2006, PASJ, 58, 1023

Sadakane, K., \& Nishida, M. 1986, PASP, 98, 685

Santos, N. C., Lovis, C., Pace, G., Melendez, J., \& Naef, D. 2009, A\&A, 493, 309

Schmidt-Kaler, T. 1982, Landolt-Börnstein: Group IV, Vol. 2b, Stars and Star Clusters (Berlin: Springer)

Schuster, W. J., \& Nissen, P. E. 1986, IBVS, 2943, 1

Shao, M., \& Colavita, M. M. 1992, ARA\&A, 30, 457

Song, I., Caillault, J.-P., Barrado y Navascués, D., \& Stauffer, J. R. 2001, ApJ, 546,3

Soummer, R., Brendan Hagan, J., Pueyo, L., et al. 2011, ApJ, 741, 55

Su, K. Y. L., Rieke, G. H., Stapelfeldt, K. R., et al. 2009, ApJ, 705, 314

Sudol, J. J., \& Haghighipour, N. 2012, ApJ, 755, 38

ten Brummelaar, T. A., McAlister, H. A., Ridgway, S. T., et al. 2005, ApJ, 628,453

Torres, C. A. O., Quast, G. R., Melo, C. H. F., \& Sterzik, M. F. 2008, in Handbook of Star Forming Regions, Vol. II, ed. B. Reipurth (Astronomical Society of the Pacific Monograph Publications), 757

Turcotte, S., \& Charbonneau, P. 1993, ApJ, 413, 376

van Belle, G. T., Ciardi, D. R., ten Brummelaar, T., et al. 2006, ApJ, 637, 494

van Leeuwen, F. 2007, A\&A, 474, 653

Venn, K. A., \& Lambert, D. L. 1990, ApJ, 363, 234

Yi, S., Demarque, P., Kim, Y.-C., et al. 2001, ApJS, 136, 417

Zerbi, F. M., Rodríguez, E., Garrido, R., et al. 1999, MNRAS, 303, 275

Zuckerman, B., Rhee, J. H., Song, I., \& Bessell, M. S. 2011, ApJ, 732, 61

Zuckerman, B., \& Song, I. 2004, ApJ, 603, 738 